ARTICLE

DOI: $10.1038 / \mathrm{s} 41467-018-05474-0$

\title{
Dual-template engineering of triple-layered nanoarray electrode of metal chalcogenides sandwiched with hydrogen-substituted graphdiyne
}

\author{
Sifei Zhuo ${ }^{1}$, Yusuf Shi ${ }^{1}$, Lingmei Liu $^{2}$, Renyuan $\mathrm{Li}^{1}$, Le Shi ${ }^{1}$, Dalaver H. Anjum ${ }^{3}$, Yu Han (D) $^{2}$ \& Peng Wang ${ }^{1}$
}

Hybrid nanostructures integrating electroactive materials with functional species, such as metal-organic frameworks, covalent organic frameworks, graphdiyne etc., are of significance for both fundamental research and energy conversion/storage applications. Here, hierarchical triple-layered nanotube arrays, which consist of hydrogen-substituted graphdiyne frameworks seamlessly sandwiched between an outer layer of nickel-cobalt co-doped molybdenum disulfide nanosheets and an inner layer of mixed cobalt sulfide and nickel sulfide $\left(\mathrm{Co}_{9} \mathrm{~S}_{8} /\right.$ $\mathrm{Ni}_{3} \mathrm{~S}_{2}$ ), are directly fabricated on conductive carbon paper. The elaborate triple-layered structure emerges as a useful hybrid electrode for energy conversion and storage, in which the organic hydrogen-substituted graphdiyne middle layer, with an extended $\pi$-conjugated system between the electroactive nanomaterials, provides built-in electron and ion channels that are crucial for performance enhancement. This dual-template synthetic method, which makes use of microporous organic networks to confine a self-template, is shown to be versatile and thus provides a promising platform for advanced nanostructure-engineering of hierarchical multi-layered nanostructures towards a wide range of electrochemical applications.

\footnotetext{
${ }^{1}$ Water Desalination and Reuse Center, Biological and Environmental Science and Engineering Division, King Abdullah University of Science and Technology, Thuwal 23955-6900, Saudi Arabia. ${ }^{2}$ Advanced Membranes and Porous Materials Center, Physical Sciences and Engineering Division, King Abdullah University of Science and Technology, Thuwal 23955-6900, Saudi Arabia. ${ }^{3}$ Imaging and Characterization Core Lab, King Abdullah University of Science and Technology, Thuwal 23955-6900, Saudi Arabia. Correspondence and requests for materials should be addressed to Y.H. (email: yu.han@kaust.edu.sa) or to P.W. (email: peng.wang@kaust.edu.sa)
} 
H ollow nanomaterials with functional shells and inner voids are promising for advanced energy conversion and storage applications ${ }^{1-5}$. Recently, a variety of multilayered hollow nanomaterials of metal-organic frameworks $(\mathrm{MOFs})^{6}$, carbon nanospheres ${ }^{7}$, organosilica $^{8}$, metal oxides ${ }^{9}$, sulfides $^{10,11}$, and phosphides ${ }^{12}$ have been shown to outperform the conventional fully solid and single-shelled counterparts and show potential for contributing to the sustainable development of society. For example, multi-shelled hollow nanospheres ${ }^{13,14}$, nanopolyhedra ${ }^{15,16}$, and nanotubes ${ }^{17}$ are able to deliver enhanced electrochemical activities in Li-ion batteries and water splitting compared with their single-shelled counterparts. However, up to now, the multi-shells of these hollow nanomaterials are restricted to the same chemical composition ${ }^{6-8,10-14,17}$ or reduplicative geometric morphology of yolk-shell structure ${ }^{12-16}$, which limits their potential applications. Recently, integration of electroactive nanomaterials with functional species such as $\mathrm{MOFs}^{18,19}$, covalent organic frameworks $(\mathrm{COFs})^{20}$, complexes ${ }^{21}$, graphdiyne ${ }^{22}$, and carbon ${ }^{23-30}$ etc., have experienced rapid development and have been widely applied in electrochemical-based catalysis and energy storage. In typical electrochemical applications, wellaligned nanoarrays directly grown on current collectors without any polymeric binder are considered as favorable electrode materials ${ }^{31-34}$. Furthermore, hybrid nanoarrays with multicompositions show enhanced performance owing to synergistic functions ${ }^{31,35-37}$. Although various hollow hybrid nanoarrays have been synthesized, they are mainly single- and double-shelled nanostructures with all-inorganic components ${ }^{33,35,37}$. So far, the synthesis of hierarchical triple-layered hollow hybrid nanoarrays that have different layer geometric morphologies and chemical compositions and that can be directly used as electrode material, although attractive, does not exist at the moment to the best of our knowledge.

Self-templating approaches, in which sacrificial templates are involved, are widely used in controllable fabrication of complex hollow nanostructures due to the availability of various sacrificial templates $31,34,38-43$. However, mainly due to the paucity of suitable self-templates, this approach has not been successful in synthesizing triple-layered hollow nanostructures in which each shell has different compositions and morphologies. On the other hand, diverse microporous organic networks (MONs) with great chemical and thermal stability have recently been prepared through various chemical coupling reactions ${ }^{44-49}$. Cross-linked carbon-rich frameworks of MONs, such as hydrogen-substituted graphydiyne (HsGDY) with a highly extended $\pi$-conjugated system, have shown satisfactory conductivity and ion diffusion, leading to application in flexible electrodes for $\mathrm{Li} / \mathrm{Na}$ storage $\mathrm{e}^{50}$. In addition, the applications of HsGDY have been gradually extended to organic catalysis $^{51,52}$ and photocatalysis ${ }^{53}$. Recently, it has been reported that HsGDY is able to form a rigid conformal microporous coating layer on the surface of many self-templating materials due to the robustness and well-defined porosity of $\mathrm{HsGDY}^{51}$. The HsGDY conformal coating layer is expected to have selective species permeability. Therefore, a self-template with a conformally coated HsGDY layer would permit the migration of species from the inner self-template region through the HsGDY and into the external surface of the HsGDY layer where they can be designed to react with desirable species in bulk solution and vice versa. Therefore, it is expected that an HsGDY-coated self-template is an interesting platform to synthesize triple-layered nanostructures. In this case, both the HsGDY coating layer and the inner confined self-template serve the role of template and can be considered as dual-template. The dual-template strategy that involves a HsGDY-coated self-template has the potential for producing triple-layered nanoarrays with specific chemical compositions and morphologies for each layer.
Using electricity that is generated by a photovoltaic cell as an energy source for electrocatalytic water splitting is considered to be a clean-energy technique for hydrogen production. In pursuit of a cost-effective high-performance electrocatalyst for the hydrogen evolution reaction (HER), new nanomaterial design concepts and strategies have attracted considerable efforts ${ }^{54,55}$.

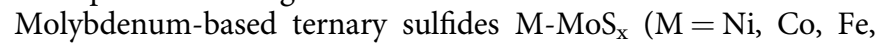
$\mathrm{Zn}, \mathrm{Cu}$ ) have been identified as promising electrocatalysts for water splitting under a wide range of $\mathrm{pH}$ values due to a synergistic effect between $\mathrm{M}$ and $\mathrm{Mo}^{56-61}$. A number of theoretical and experimental studies indicate that doping heteroatoms into the $\mathrm{MoS}_{2}$ matrix stimulates the intrinsic activity of in-plane $\mathrm{S}$ atoms ${ }^{56,57,60,61}$. Recently, integration of $\mathrm{MoS}_{2}$ with other functional materials, such as carbon ${ }^{62}$, reduced graphene oxide $(\mathrm{r}-\mathrm{GO})^{63}$, metal complexes ${ }^{21}$, and other transition metal sulfides $36,64,65$, has led to enhanced HER performance. The availability of the various $\mathrm{Ni}$ - and Co-based nanostructures makes them promising candidates for the construction of hierarchical nanostructures, along with $\mathrm{MoS}_{2}$, for enhanced electrocatalytic performance. Thus, the integration of multiple electroactive metal chalcogenides with HsGDY, especially within a multi-layered nano-framework directly on a conductive substrate, is highly desirable for HER-based applications.

Here, we provide a proof-of-concept of using an HsGDYcoated self-template as a dual template to fabricate triple-layered nanostructures. We demonstrate the synthesis of hierarchical triple-layered nanoarrays of transition metal chalcogenides (TMCs) directly on a carbon paper by using HsGDY-coated nickel cobalt hydroxyl carbonate $(\mathrm{NiCoHC})$ nanowire arrays as a dual template. The synthesized material has built-in HsGDY nanolayers sandwiched between Ni-, Co-co-doped $\mathrm{MoS}_{2}(\mathrm{Ni}, \mathrm{Co}-$ $\left.\mathrm{MoS}_{2}\right)$ nanosheets, and mixed $\mathrm{NiCoS}\left(\mathrm{Co}_{9} \mathrm{~S}_{8}, \mathrm{Ni}_{3} \mathrm{~S}_{2}\right)$ nanotubes. The synthesis involves preparation of $\mathrm{NiCoHC}$ nanowire arrays as a bimetallic self-template, confinement of the NiCoHC nanowire arrays with conformally coated HsGDY layers (NiCoHC@HsGDY), and transformation of NiCoHC@HsGDY into tri-layered NiCoS@HsGDY@Ni,Co- $\mathrm{MoS}_{2}$ (Fig. 1). The microporous HsGDY layer (step II) in NiCoHC@HsGDY acts as not only a physical separator to confine the in-situ conversion of $\mathrm{NiCoHC}$ nanowires to mixed $\mathrm{NiCoS}$ nanotubes in the interior, but also a chemical nucleation platform with an ion channel to generate $\mathrm{Ni}, \mathrm{Co}-\mathrm{MoS}_{2}$ nanosheets on the external surface (step III). This triple-layered nanoarray on carbon paper incorporates inorganics (i.e., TMCs) with organics (i.e., HsGDY), and the intermediate HsGDY layer can work as a built-in electron and ion channel owing to the highly extended $\pi$-conjugated system, all pointing toward a potential for being used directly as a binderfree and self-supported electrode. Due to the hierarchical nanostructures, the enhanced number of active sites, improved electron conductivity and mass transport of the geometric constraint, the material shows high current density, low overpotential, and excellent anti-aggregation behaviors over a wide $\mathrm{pH}$ range $(0-14)$ in HER. The dual-template approach is further extended to prepare other triple-layered hollow nanostructures of

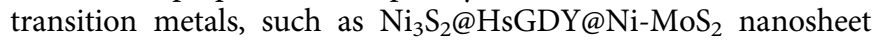
arrays and $\mathrm{Co}_{9} \mathrm{~S}_{8} @ \mathrm{HsGDY} @ \mathrm{Co}-\mathrm{MoS}_{2}$ nanotube arrays, proving its versatility.

\section{Results}

Construction of the dual-template. In this work, the conformally coated microporous HsGDY layers are the key to the dualtemplate approach. It serves not only as a conformal separator to confine the self-template, but also acts as a mass transport channel and rigid scaffold for the nucleation and growth of the third layer on the external surfaces. In detail, well-aligned 


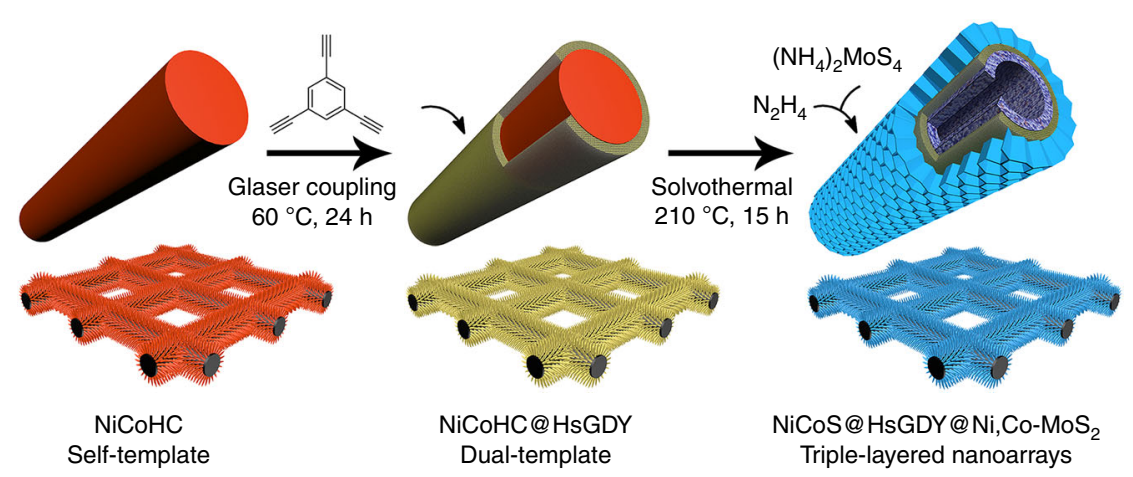

Fig. 1 Schematic illustration of the fabrication of triple-layered nanotube arrays. The chemical synthesis of hierarchical triple-layered NiCoS@HsGDY@Ni,

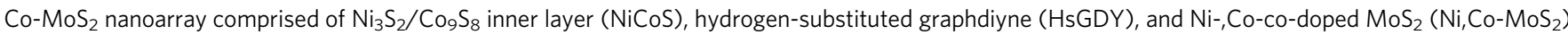
is achieved with a $\mathrm{HsGDY}$-coated $(\mathrm{Ni}, \mathrm{Co})(\mathrm{OH})_{2} \mathrm{CO}_{3}(\mathrm{NiCoHC})$ nanoarray as a dual template

$\mathrm{NiCoHC}$ nanowire arrays are first prepared directly on carbon fibre paper in virtue of the bimetallic character and availability of the various $\mathrm{Ni}$ - and Co-based nanostructures ${ }^{32}$. As shown in the scanning electron microscopy (SEM) images (Fig. 2a, b and Supplementary Fig. 1), well-aligned tapering nanowire arrays with diameters of $\sim 100-200 \mathrm{~nm}$ and lengths up to $2 \mu \mathrm{m}$ are uniformly distributed on the carbon paper. The radial growth of these nanowires directly around the carbon fibres and the tapering character of each nanowire leads to increased surface area and thus are potentially beneficial for many applications. The unimodal profile and uniform spatial distribution of elemental $\mathrm{Ni}$, $\mathrm{Co}, \mathrm{O}$, and $\mathrm{C}$, as shown in the line-scan electron energy loss spectroscopy (EELS) profiles (Fig. 2c) and scanning transmission electron microscopy-electron energy loss spectroscopy (STEMEELS) elemental mapping (Supplementary Fig. 2d), respectively, indicate the solid nature of these nanowires with bimetallic characteristics. The characteristic vibration and diffraction peaks shown in the Fourier transform infrared (FTIR) spectra (Supplementary Fig. 7) and X-ray diffraction (XRD) patterns (Supplementary Fig. 3), respectively, further identify these nanoarrays as hydroxyl-carbonate, $(\mathrm{Ni}, \mathrm{Co})(\mathrm{OH})_{2} \mathrm{CO}_{3}$, with high crystallinity ${ }^{32,37}$. Inductively coupled plasma-atomic emission spectrometry (ICP-AES) reveals the atomic ratio of $\mathrm{Co} / \mathrm{Ni}$ as about 2 , which indicates the composition of $\mathrm{NiCoHC}$ as $\mathrm{Ni}_{0.67} \mathrm{Co}_{1.33}(\mathrm{OH})_{2} \mathrm{CO}_{3}$. These results demonstrate that bimetallic $\mathrm{NiCoHC}$ nanowire arrays have been successfully fabricated as the self-template.

Then, a layer of HsGDY is directly coated onto each NiCoHC nanowire to form a coating by Glaser coupling of 1,3,5triethynylbenzene ${ }^{51}$. As shown in the SEM (Fig. 2d, e and Supplementary Fig. 4) and transmission electron microscopy (TEM) images (Fig. 2f and Supplementary Fig. 5a-c), the HsGDY on each nanowire inherits well-aligned and tapering character of the underlying self-template of the NiCoHC nanowire, indicating the seamless conformal coating of HsGDY. As shown in Fig. 2f, a thin and uniform layer perfectly covers and preserves the underlying morphology of $\mathrm{NiCoHC}$ at the right corner. The thickness of the HsGDY coating is around $10 \mathrm{~nm}$. The straight boundaries between the crystalline $\mathrm{NiCoHC}$ nanowires and HsGDY layers are clearly distinguishable and further confirm the conformal coating nature of the HsGDY layers (Fig. 2g). The XRD patterns of NiCoHC do not show any changes before and after HSGDY coating (Supplementary Fig. 3), which is consistent with TEM observations. Obviously, the corresponding elemental distribution of $\mathrm{C}$, with wider coverage as compared with $\mathrm{Ni}, \mathrm{Co}$, and $\mathrm{O}$ (Fig. 2h, i and Supplementary Fig 5e), supports the full and uniform coating of $\mathrm{NiCoHC}$ nanowires by the HsGDY.
To ascertain the chemical composition and porosity of the HsGDY coatings, some discrete HsGDY nanotubes are intentionally fabricated by removing the $\mathrm{NiCoHC}$ by acid etching process, which would otherwise affect the ${ }^{13} \mathrm{C}$ nuclear magnetic resonance (NMR) analysis due to the presence of paramagnetic species $\left(\mathrm{Co}^{2+}\right)$ and interfere with the $\mathrm{N}_{2}$ adsorption/desorption (Supplementary Fig. 6). The isolated HsGDY sample is characterized using NMR, X-ray photoelectron spectroscopy (XPS), Raman, and FTIR spectra. The solid-phase ${ }^{13} \mathrm{C}$ NMR spectrum (Fig. 3b) shows the signals at $\delta=136.2 \mathrm{ppm}$ and 123.1 ppm assigned to the $s p^{2}$-hybridized aryl and peaks at $\delta=90.3$ ppm and $81.5 \mathrm{ppm}$, which can be ascribed to the $s p$-hybridized alkyne, and thus indicates the highly conjugated electronic structure of the as-prepared HsGDY layers with extended $\pi$ conjugation system bearing $s p$ - and $s p^{2}$-hybridized carbon atoms $50,51,66$. This result is in accordance with the XPS results (vide infra) with sub-peaks at 284.3 and $285 \mathrm{eV}$ attributed to the $\mathrm{C} 1 s$ orbital of $\mathrm{C}=\mathrm{C}\left(s p^{2}\right)$ and $\mathrm{C} \equiv \mathrm{C}(s p)$, respectively ${ }^{50,67}$. Besides, the peaks of $1370 \mathrm{~cm}^{-1}$ and $1597 \mathrm{~cm}^{-1}$ of the Raman spectrum correspond to the $\mathrm{D}$ band and $\mathrm{G}$ band of vibration of $s p^{2}$ carbon domains in aromatic rings, respectively (Fig. 3c) ${ }^{67}$, and the weak peaks located at $2327 \mathrm{~cm}^{-1}$ can be assigned to the alkyne-related stretching vibrations, revealing the large $\pi$ conjugation system of HsGDY cross-links aromatic rings with acetylenic bonds ${ }^{50}$. Furthermore, the representative vibration peaks for $\mathrm{C} \equiv \mathrm{C}$ bond $\left(2190 \mathrm{~cm}^{-1}\right)$ and aromatic $\mathrm{C}-\mathrm{H}\left(881 \mathrm{~cm}^{-1}\right.$, $3060 \mathrm{~cm}^{-1}$ ) in the FTIR spectrum (Supplementary Fig. 7) identify the structure of HsGDY with $s p$ - and $s p^{2}$-hybridized carbon atoms as poly(phenylene butadiynylene) ${ }^{50,52}$. In addition, the analysis of the $\mathrm{N}_{2}$ isotherms (Fig. 3d) based on the Brunauer-Emmett-Teller (BET) theory indicates that the HsGDY layers possess micropores with diameters around $1.2 \mathrm{~nm}$ and a surface area up to $651 \mathrm{~m}^{2} \mathrm{~g}^{-1}$. Therefore, coreshell nanowire arrays comprised of a $\mathrm{NiCoHC}$ nanowires core and a conformal surface coating of rigid and microporous HsGDY as the shell are successfully achieved. The conformal and uniform coating of HsGDY on each self-template nanowire with coaxial character enable its use as a dual-template platform for the homogeneous growth of a third layer right on top of the HsGDY coating layer.

Synthesis of the triple-layered nanotube arrays. These asprepared dual-template of NiCoHC@HsGDY are utilized to construct molybdenum-based ternary sulfides by reaction with $\mathrm{NH}_{4} \mathrm{MoS}_{4}$ in the presence of $\mathrm{N}_{2} \mathrm{H}_{4}$. The SEM images show, after the reaction, the well-aligned structure of the self-templating nanowires are preserved (Fig. 4a) with each nanowire fully 


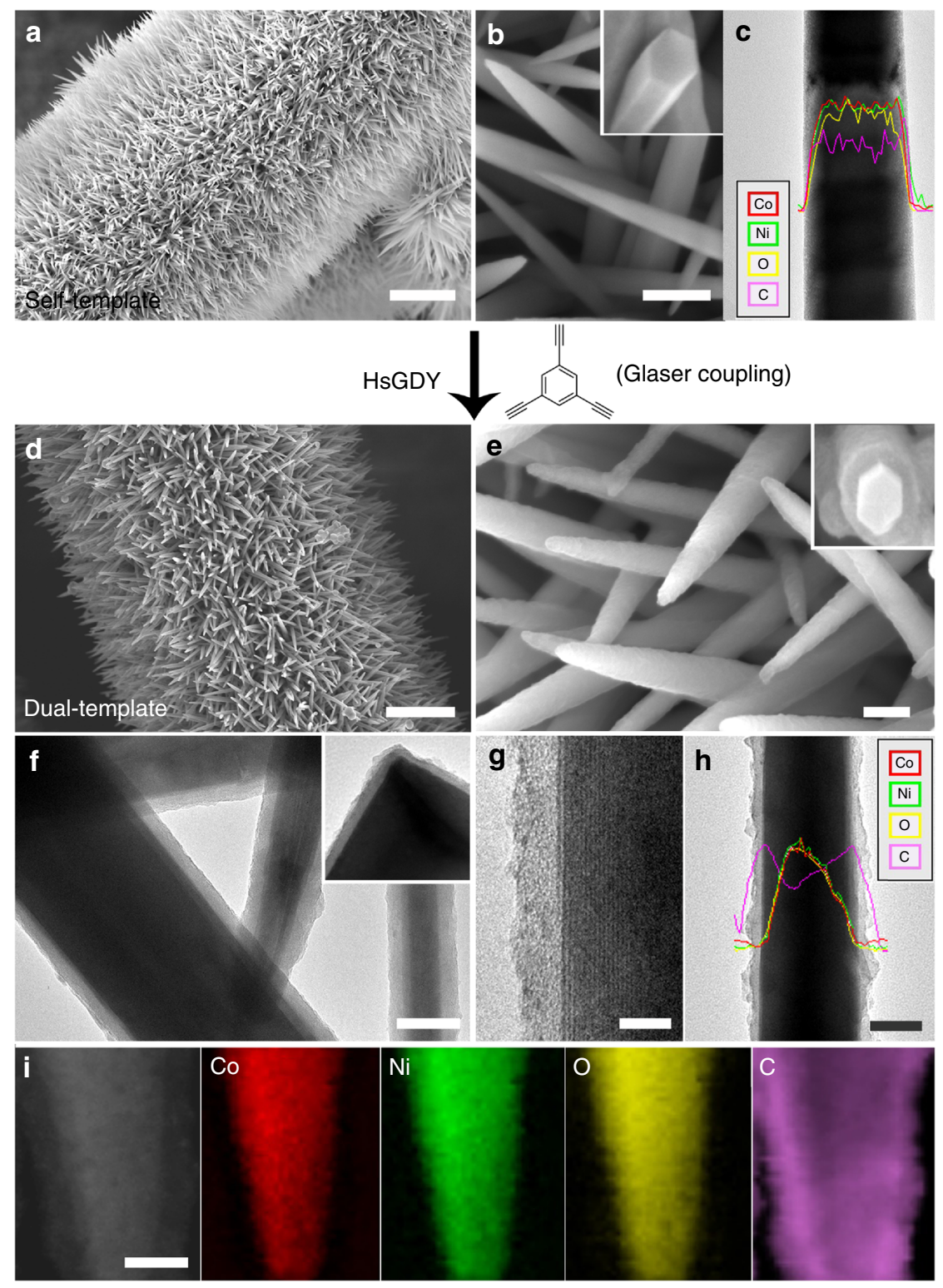

Fig. 2 Scanning electron microscopy and transmission electron microscopy micrographs. a, b Scanning electron microscopy (SEM) images, c Transmission electron microscopy (TEM) image and line-scan electron energy loss spectroscopy $(\mathrm{EELS})$ elemental distributions of the $(\mathrm{Ni}, \mathrm{Co})(\mathrm{OH})_{2} \mathrm{CO} \mathrm{O}_{3}(\mathrm{NiCoHC})$ nanowires arrays. d, e SEM images, $\mathbf{f}, \mathbf{g}$ TEM images, $\mathbf{h}$ line-scan EELS elemental distributions, and $\mathbf{i}$ Scanning transmission electron microscopy (STEM)EELS elemental mapping of the NiCoHC@hydrogen-substituted graphdiyne (HsGDY) nanowires. The insets of $\mathbf{b}, \mathbf{e}$, and $\mathbf{f}$ are the corresponding SEM images of the cross-sections and TEM image of the corner, respectively, taking from some broken nanofibres. Scale bars: (a, d) $2 \mu \mathrm{m},(\mathbf{b}, \mathbf{e}) 200 \mathrm{~nm}$, $(\mathbf{f}, \mathbf{h}, \mathbf{i}) 50 \mathrm{~nm},(\mathbf{g}) 10 \mathrm{~nm}$

covered with interconnected nanosheets (Fig. $4 \mathrm{~b}$ and Supplementary Fig. 8). Close observation by TEM (Fig. 4c) and high magnification SEM (inset of Fig. $4 \mathrm{~b}$ and Supplementary Fig. 8) at broken areas of the samples clearly reveal a triple-layered tube-intube nanostructure. High angle annular dark field-scanning transmission electron microscopy (HAADF-STEM) (Fig. 4d) confirms that the triple-layered structures are composed of an HsGDY middle layer seamlessly sandwiched between a flowerlike and loosely assembled nanosheet outer layer and a hollowedout nanotube inner layer. Impressively, the clear-cut and continuous layer boundaries on both sides of the HsGDY middle layer (Fig. 4d) suggest the firmly confined inner layer and the free growing outer layer. The exterior flower-like assembled nanosheets with well exposed edges and the interior hollowed-out nanotubes would greatly enhance the number of active sites in these triple-layer structures. High-resolution TEM (HRTEM) images and energy dispersive spectroscopy (EDS) are then collected to analyze elemental compositions of these three layers one by one. First, the outer layer of the nanosheets show an expanded interplanar spacing (e.g., $0.65 \mathrm{~nm}$ and $0.68 \mathrm{~nm}$ ) and lattice distance of $0.27 \mathrm{~nm}$ (Fig. 4e and Supplementary Fig. 9), which are assigned solely to hexagonal $\mathrm{MoS}_{2}\left(2 \mathrm{H}-\mathrm{MoS}_{2}\right)$. The corresponding EDS spectrum reveals the presence of $\mathrm{Ni}$ and $\mathrm{Co}$ in the nanosheets (Supplementary Fig. 10), implying the incorporation of $\mathrm{Ni}$ and $\mathrm{Co}$ ions, thus insinuating outward diffusion of $\mathrm{Ni}$ and Co ions through HsGDY layers ${ }^{57}$. The uniform distribution of elemental $\mathrm{Ni}, \mathrm{Co}, \mathrm{Mo}$, and $\mathrm{S}$ with encapsulated elemental $\mathrm{C}$ with distinguishable contrast in the side-view STEM-EELS elemental mapping (Fig. 4g) further identify the incorporation of $\mathrm{Ni}$ and $\mathrm{Co}$ ions within $\mathrm{MoS}_{2}$. Therefore, it is believed that the outer layer of the triple-layered structure is Ni-, Co-co-doped $\mathrm{MoS}_{2}$, denoted as $\mathrm{Ni}, \mathrm{Co}-\mathrm{MoS}_{2}$ hereafter. On the other hand, the hollowed-out 
a

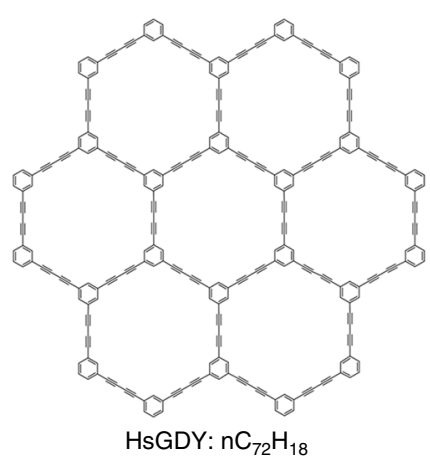

C

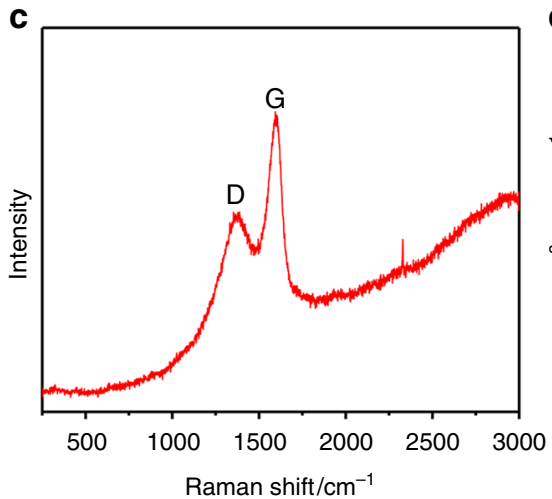

b

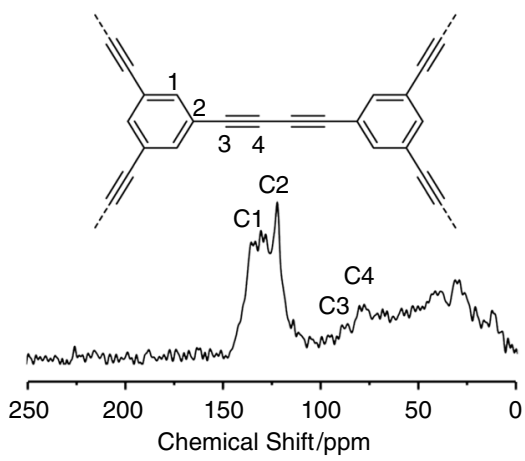

d

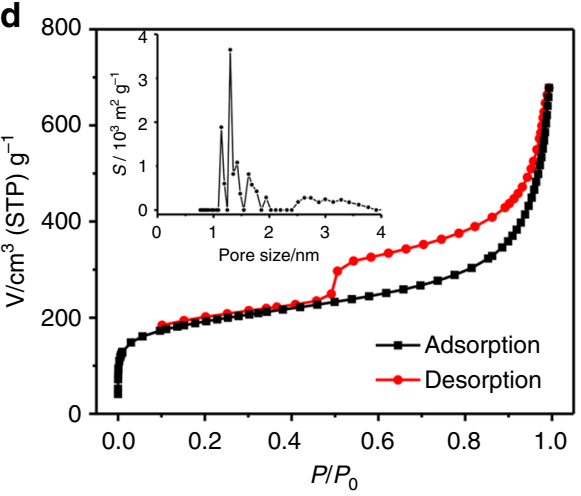

Fig. 3 Structural characterizations of hydrogen-substituted graphdiyne. a Schematic illustration, $\mathbf{b}$ solid ${ }^{13} \mathrm{C}$ nuclear magnetic resonance (NMR) spectrum, c Raman spectrum, and $\mathbf{d} \mathrm{N}_{2}$ adsorption/desorption isotherms of the discerte conformal coated hydrogen-substituted graphdiyne (HsGDY). The inset of d is the corresponding pore size distribution diagram

nanotubes are identified as $\mathrm{Co}_{9} \mathrm{~S}_{8}$ with lattice distance of 0.175 $\mathrm{nm}(440)$ and $0.30 \mathrm{~nm}(311)$, and $\mathrm{Ni}_{3} \mathrm{~S}_{2}$ with lattice distance of $0.287 \mathrm{~nm}$ (110) and $0.238 \mathrm{~nm}$ (003) under HRTEM images (Fig. 4f and Supplementary Fig. 9) ${ }^{68,69}$. To further investigate the compositions of the inside layers, ultrathin sections perpendicular to the concentric shaft are prepared using ultramicrotome to cut the sample. As shown in Fig. 4h, the triple-layered structure is apparent by the onion-like structure of the cross-section. Notably, only $\mathrm{C}$ is detected in the middle layer, which suggests that no TMC residues block the micropores of the HsGDY layers. Furthermore, the elements of $\mathrm{Ni}, \mathrm{Co}$, and $\mathrm{S}$ distribute over both the inner and outer layers, while Mo dominantly accumulates around the outer layer, indicating that mixed $\mathrm{NiCoS}\left(\mathrm{Ni}_{3} \mathrm{~S}_{2}, \mathrm{Co}_{9} \mathrm{~S}_{8}\right)$ dominates the inner layer. The results so far support the successful fabrication of triple-layered nanoarrays. Efforts are then made to ascertain the detailed chemical compositions of each layer.

XRD, Raman, and XPS are further conducted to evaluate the detailed elemental compositions and chemical states of the triplelayered nanostructure. The well-resolved diffraction XRD peaks of cubic $\mathrm{Co}_{9} \mathrm{~S}_{8}$ (JCPDS No.86-2273) ${ }^{65}$ are clearly seen, while the peaks of $\mathrm{Ni}_{3} \mathrm{~S}_{2}$ and $\mathrm{MoS}_{2}$ are weak (Fig. 5a). However, Raman spectra (Fig. 5b and Supplementary Fig. 11) reveal the presence of $\mathrm{Ni}_{3} \mathrm{~S}_{2}\left(297 \mathrm{~cm}^{-1} \text { and } 341 \mathrm{~cm}^{-1}\right)^{68}, \mathrm{Co}_{8} \mathrm{~S}_{9}\left(463 \mathrm{~cm}^{-1}, 507 \mathrm{~cm}^{-1}\right.$ and $\left.651 \mathrm{~cm}^{-1}\right)^{69}, \mathrm{Ni}, \mathrm{Co}-\mathrm{MoS}_{2}$, and HsGDY in the triple-layered nanostructure. The increased ID/IG value of HsGDY after the chemical transformation is attributed to the defects formed in the HsGDY during the solvothermal treatment process (Supplementary Fig. 12) and the peaks located at $817 \mathrm{~cm}^{-1}, 882 \mathrm{~cm}^{-1}$, and $933 \mathrm{~cm}^{-1}$ can be assigned to $\mathrm{Ni}$ - and Mo-based oxides that are easily generated by laser irradiation during the Raman test ${ }^{70}$. Notably, the Raman bands at $379 \mathrm{~cm}^{-1}$ and $401 \mathrm{~cm}^{-1}$ correspond to the in-plane of $E_{2 g}$ and out-of-plane of $A_{1 g}$ modes of hexagonal
$\mathrm{MoS}_{2}$, indicating the similar layered structure of $\mathrm{Ni}$-, Co-co-doped $\mathrm{MoS}_{2}$ with $\mathrm{MoS}_{2}{ }^{56}$. The XPS survey spectrum confirms the presence of $\mathrm{Ni}, \mathrm{Co}, \mathrm{Mo}, \mathrm{S}$, and $\mathrm{C}$ with specific chemical states (Fig. 5c). In the high-resolution XPS spectrum of Ni 2p (Fig. 5d), the binding energies (BEs) at $855.7 \mathrm{eV}$ and $873.4 \mathrm{eV}$ with a spin energy of $17.7 \mathrm{eV}$ are ascribed to $\mathrm{Ni}^{2+} 2 p_{3 / 2}$ and $\mathrm{Ni}^{2+} 2 p_{1 / 2}$, respectively, and the BEs of $\mathrm{Ni}^{0} 2 p$ shown in $852.9 \mathrm{eV}$ and 870.3 $\mathrm{eV}$ confirm the formation of $\mathrm{Ni}_{3} \mathrm{~S}_{2}{ }^{68,71}$. The high-resolution XPS of Co $2 p$ (Fig. $5 \mathrm{e}$ ) can be deconvoluted into four primary peaks at $778.5 \mathrm{eV}\left(\mathrm{Co}^{3+}\right), 780.8 \mathrm{eV}\left(\mathrm{Co}^{2+}\right)$ for $\mathrm{Co} 2 p_{3 / 2}$, and $793.6 \mathrm{eV}\left(\mathrm{Co}^{3}\right.$ $\left.{ }^{+}\right), 797.0 \mathrm{eV}\left(\mathrm{Co}^{2+}\right)$ for Co $2 p_{1 / 2}$, respectively, which could be assigned to $\mathrm{Co}_{8} \mathrm{~S}_{9}{ }^{69}$. Specially, two sharp peaks located at 228.4 $\mathrm{eV}$ and $231.85 \mathrm{eV}$, attributed to Mo $3 d_{5 / 2}$ and Mo $3 d_{3 / 2}$, are also clearly observed, indicating the dominance of $\mathrm{Mo}^{4+}$ in the synthesized nanostructures (Fig. 5f $)^{57}$. The presence of $\mathrm{Mo}^{6+}\left(3 d_{3 / 2}, 235.2 \mathrm{eV}\right)$ in the XPS spectrum is attributed to the following: (1) the residual $\left(\mathrm{NH}_{4}\right)_{2} \mathrm{MoS}_{4}$ species from the hydrothermal process can be adsorbed onto the surface of the tri-layered nanostructures ${ }^{57}$, and (2) partial surface oxidation in air can potentially lead to the presence of $\mathrm{Mo}^{6+}$ in these triplelayered nanostructures ${ }^{72}$. Fig. $5 \mathrm{~g}$ shows the presence of typical coordination of sulfur with metal ions, located at $161.4 \mathrm{eV}$ and $162.6 \mathrm{eV}$ for $\mathrm{S}^{2-} 2 p_{3 / 2}$ and $\mathrm{S}^{2-} 2 p_{1 / 2}$, respectively. In addition, the C $1 s$ XPS spectrum (Fig. 5h) with two sub-peaks at $284.3 \mathrm{eV}$ for $\mathrm{C}-\mathrm{C}\left(s p^{2}\right)$ and $285 \mathrm{eV}$ for C-C $(s p)$ verifies the existence of HsGDY and confirms that HsGDY is not changed during the chemical reaction ${ }^{50}$. These results verify that the synthesized triple-layered nanostructure has a chemical composition of $\mathrm{NiCoS}\left(\mathrm{Ni}_{3} \mathrm{~S}_{2}, \mathrm{Co}_{9} \mathrm{~S}_{8}\right) @ \mathrm{HsGDY} @ \mathrm{Ni}, \mathrm{Co}-\mathrm{MoS}_{2}$.

To better understand the mechanism of the transformation of NiCoHC@HsGDY nanowire arrays into the triple-layered NiCoS@HsGDY@Ni,Co- $\mathrm{MoS}_{2}$, additional control experiments are conducted. In the absence of $\mathrm{N}_{2} \mathrm{H}_{4}$, there are only $\mathrm{Ni}_{\mathrm{x}} \mathrm{Co}_{1}$ - 

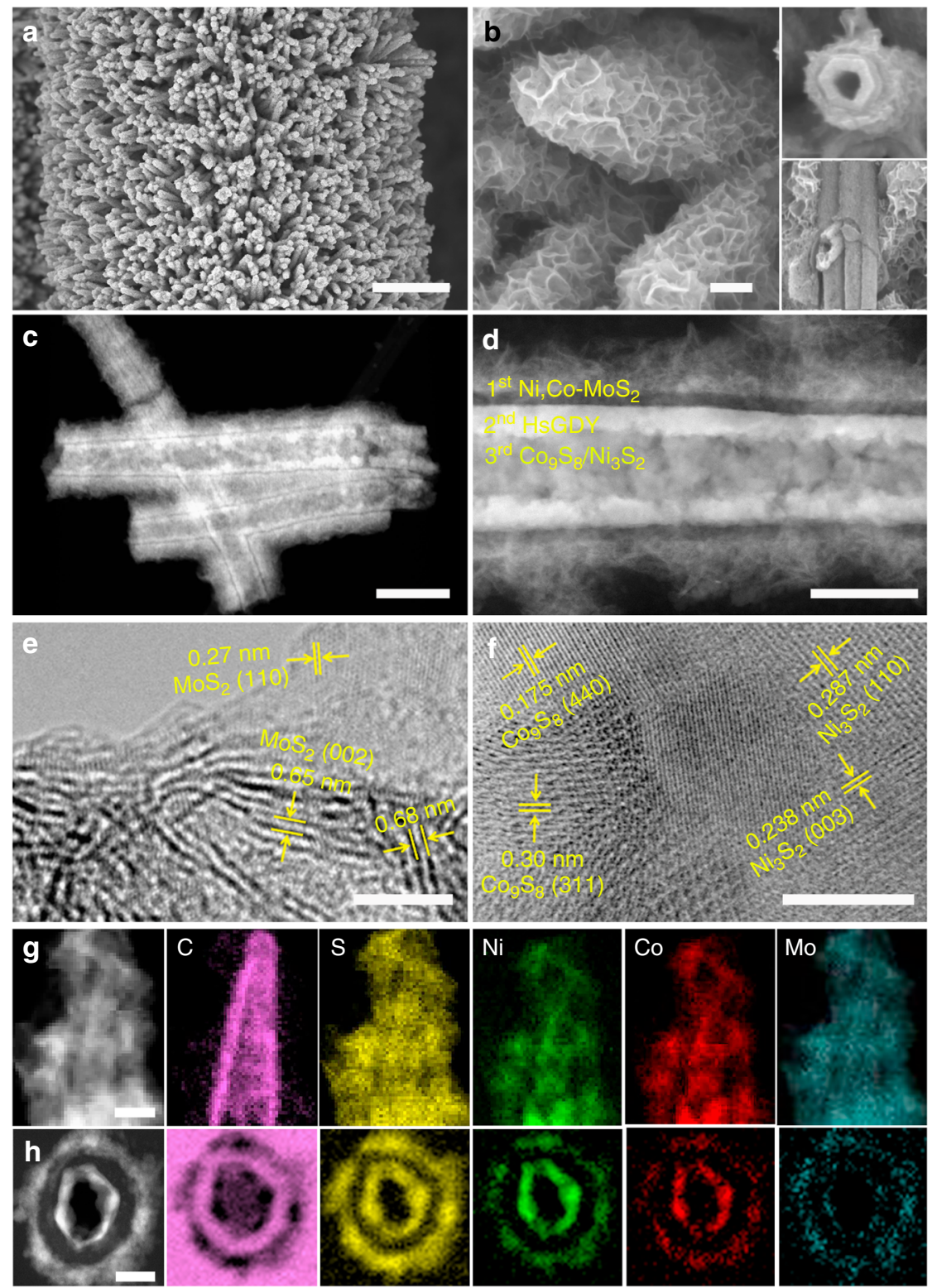

Fig. 4 Micrographs of the tri-layered nanoarrays. a, b scanning electron microscopy (SEM) images, $\mathbf{c}$, $\mathbf{d}$ high angle annular dark field-scanning transmission electron microscopy (HAADF-STEM) images, e, f high-resolution transmission electron microscopy (HRTEM) images, $\mathbf{g}$ side-view, and $\mathbf{h}$ microtomeprepared cross-section scanning transmission electron microscopy-energy loss spectroscopy (STEM-EELS) elemental mappings of the hierarchical triplelayered NiCoS@HsGDY@Ni,Co-MoS 2 nanotubular arrays comprised of $\mathrm{Ni}_{3} \mathrm{~S}_{2} / \mathrm{Co}_{9} \mathrm{~S}_{8}$ inner layer (NiCoS), hydrogen-substituted graphdiyne (HsGDY), and $\mathrm{Ni}$-,Co-co-doped $\mathrm{MoS}_{2}\left(\mathrm{Ni}, \mathrm{Co}_{-} \mathrm{MoS}_{2}\right)$. The insets of $\mathbf{b}$ show some broken areas; $\mathbf{e}$ and $\mathbf{f}$ are collected at the external $\mathrm{Ni}, \mathrm{Co}_{\mathrm{M}} \mathrm{MoS} 2$ nanosheets and the interior NiCoS nanotubes, respectively. Scale bars: a $2 \mu \mathrm{m}, \mathbf{b}, \mathbf{d} 100 \mathrm{~nm}, \mathbf{c} 200 \mathrm{~nm}$, e, f $5 \mathrm{~nm}, \mathbf{g} 50 \mathrm{~nm}, \mathbf{h} 20 \mathrm{~nm}$

${ }_{x} \mathrm{MoS}_{4}$ nanoparticles formed on the HsGDY surfaces while the inner layer disappears (Supplementary Fig. 13g-i). In the absence of the HsGDY conformal coating layer, namely, in the presence of only the self-template of NiCoHC, a mixed single-layered $\mathrm{NiCoMoS}\left(\mathrm{Co}_{9} \mathrm{~S}_{8}, \mathrm{Ni}_{3} \mathrm{~S}_{2}, \mathrm{Ni}, \mathrm{Co}-\mathrm{MoS}_{2}\right)$ nanotubes are obtained, which is convincing evidence of the critical role of the HsGDY conformal coating layer in the successful synthesis of the triplelayered nanostructure (Supplementary Fig. 13d-f). Thus, we believe that the conformal coating of HsGDY layer serves two roles: (1) physical separation with ion channels to permit two different reaction spaces on its two sides, and (2) physical embrace that keeps the inner layer firmly in place. On the one hand, $\mathrm{S}^{2-}$ generated by the decomposition of $\left(\mathrm{NH}_{4}\right)_{2} \mathrm{MoS}_{4}$ in the presence of $\mathrm{N}_{2} \mathrm{H}_{4}$ firstly diffuses inward across $\mathrm{HsGDY}$ to generate interior $\mathrm{Ni}_{3} \mathrm{~S}_{2}$ and $\mathrm{Co}_{8} \mathrm{~S}_{9}$. On the other hand, some $\mathrm{Ni}^{2+}$ and $\mathrm{Co}^{2+}$ originally in the self-template of $\mathrm{NiCoHC}$ are able to diffuse outward across the HsGDY to form Ni,Co- $\mathrm{MoS}_{2}$ on the external surface of HsGDY where they react with $\mathrm{MoS}_{4}{ }^{2-}$ in the presence of $\mathrm{N}_{2} \mathrm{H}_{4}$. In this work, further efforts are made to prove its versatility by synthesizing hollow triple-layered $\mathrm{Co}_{9} \mathrm{~S}_{8} @ \mathrm{HsG}_{\text {- }}$ DY@Co- $\mathrm{MoS}_{2}$ nanotube arrays (Fig. 6a-d) and $\mathrm{Ni}_{3} \mathrm{~S}_{2} @ \mathrm{HsG}-$ DY@Ni-MoS ${ }_{2}$ nanosheet arrays (Fig. 6e, f, Supplementary Fig. 14) using the dual-template of cobalt hydroxyl-fluoride@HsGDY (Co(OH)F@HsGDY) and nickle hydroxyl-fluoride $(\mathrm{Ni}(\mathrm{OH}) \mathrm{F} @ \mathrm{HsGDY})$, respectively. Both of the dual templates are grown directly on carbon paper and the details of these synthesis can be found in Supplementary Methods 1 .

Electrochemical performance as electrodes. Based on the conductivity and ion diffusion capability of the HsGDY middle layer 

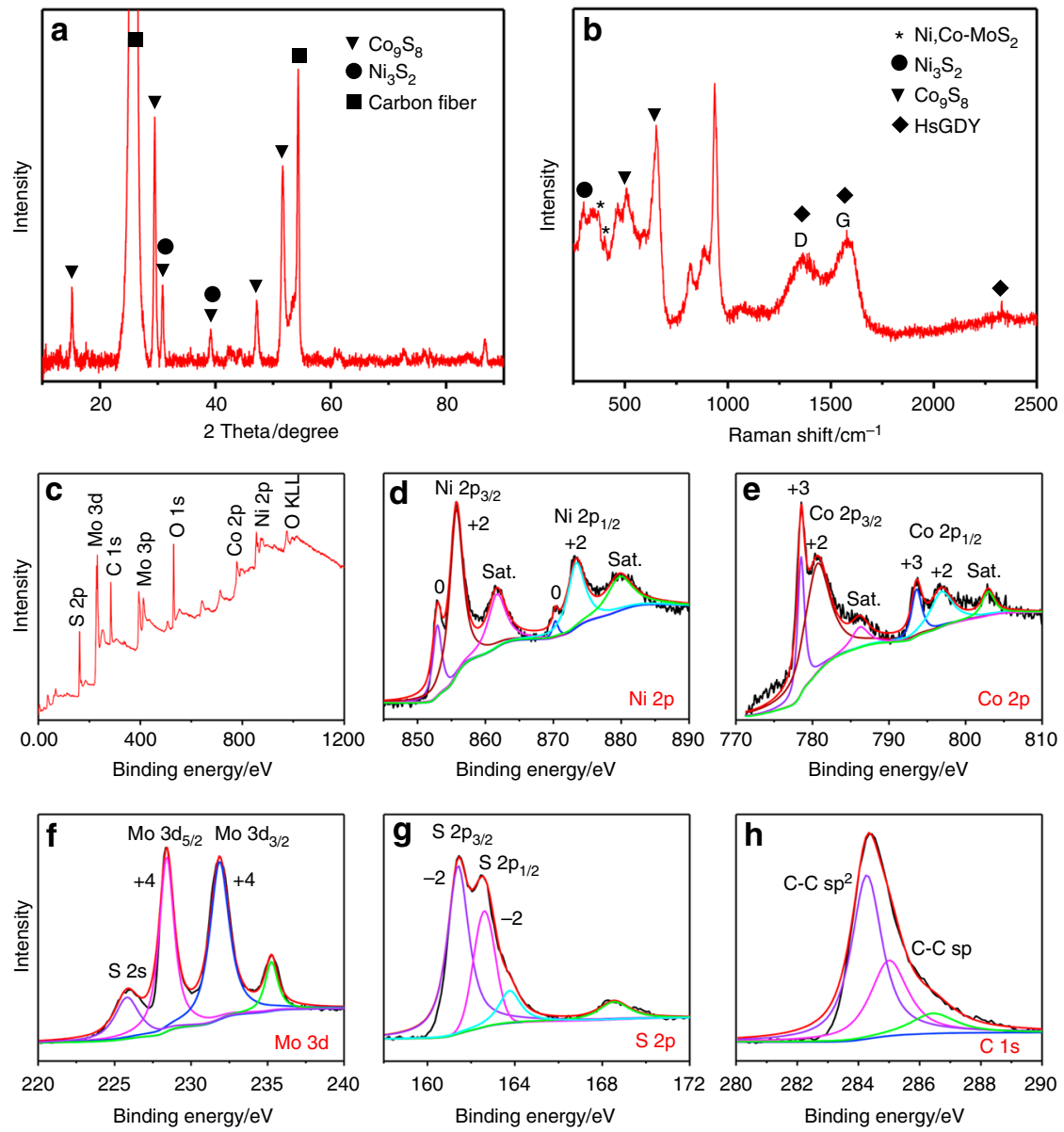

Fig. 5 Component characterizations of the tri-layered nanoarrays. a X-ray diffraction (XRD) pattern, b Raman spectrum, $\mathbf{c} X$-ray photoelectron spectroscopy (XPS) survey spectrum, and $\mathbf{d}-\mathbf{h}$ high-resolution XPS spectra of Ni, Co, Mo, S, and C for the triple-layered NiCoS@HsGDY@Ni,Co-MoS 2 nanotubes comprised of $\mathrm{Ni}_{3} \mathrm{~S}_{2} / \mathrm{Co}_{9} \mathrm{~S}_{8}$ inner layer (NiCoS), hydrogen-substituted graphdiyne (HsGDY), and Ni-,Co-co-doped $\mathrm{MoS}_{2}\left(\mathrm{Ni}, \mathrm{Co}_{-}-\mathrm{MoS}{ }_{2}\right.$ )

in the synthesized triple-layered nanostructure and the fact that the triple-layered nanostructures are grown on conductive carbon paper, the performance of the material as an electrode for HER over a wide range of $\mathrm{pH} 0-14$ is assessed. To substantiate the advantage of the tri-layered nanostructure in this work and to reveal the function of the HsGDY in the nanotube arrays (Supplementary Fig. 15), NiCoS, NiCoS@HsGDY, and mixed NiCoMoS $\left(\mathrm{Ni}_{3} \mathrm{~S}_{2}, \mathrm{Co}_{9} \mathrm{~S}_{8}, \mathrm{Ni}, \mathrm{Co}-\mathrm{MoS}_{2}\right.$ ) (Supplementary Fig. 16) are further fabricated purposefully as contrast electrodes. To directly reflect the intrinsic electrocatalytic behavior of these tri-layered nanoarrays, an $(i R)$ correction was applied to compensate the ohmic potential drop lossing from the solution resistance, i.e., the overpotential presented in Fig. 7a was corrected by $i R$, where $i$ means the current and $R$ refers to the Ohmic electrolyte resistance acquired by impedance measurements. As shown in Fig. 7a, the increase in HER performance from NiCoS, to NiCoS@HsGDY and further to NiCoS@HsGDY@Ni,CoMoS 2 indicates the role of each layer and the necessity of the tri-layered structure. More specifically, NiCoS@HsGDY@Ni,Co-MoS 2 delivers an overpotential of $124 \mathrm{mV}$ with a Tafel slop of $64.3 \mathrm{mV} \mathrm{dec}^{-1}$ at a current density of $10 \mathrm{~mA} \mathrm{~cm}^{-2}$, which compares favorably against the NiCoMoS (132 mV with Tafel slop of $73.7 \mathrm{mV} \mathrm{dec}^{-1}$ ), NiCoS@HsGDY (197 mV with Tafel slop of $64.7 \mathrm{mV} \mathrm{dec}^{-1}$ ) and $\mathrm{NiCoS}\left(224 \mathrm{mV}\right.$ with Tafel slop of $\left.72.5 \mathrm{mV} \mathrm{dec}^{-1}\right)$. The enhanced HER performance of NiCoS@HsGDY over NiCoS points to the beneficial role of the HsGDY layers serving as selective electron and ion channel allowing electrolyte access to the inner layer. The further enhanced activity of NiCoS@HsGDY@Ni,Co- $\mathrm{MoS}_{2}$ compared to NiCoS@HsGDY indicates the importance of having three layers with different compositions and structures. In addition, the electrochemical impedance spectroscopy (EIS) spectra show that NiCoS@HsGDY@Ni,Co-MoS 2 and NiCoS@HsGDY have smaller charge transfer resistance than NiCoMoS and NiCoS, respectively, at an overpotential of $200 \mathrm{mV}$ (Fig. 7c). As known, the solution resistance $R_{\mathrm{s}}$ is independent of overpotential of HER, while the charge transfer resistance $R_{\mathrm{ct}}$ determines the electrocatalytic kinetics of HER. As displayed in the equivalent circuit diagrams (Supplementary Fig. 17), the values of $R_{\mathrm{ct}}$ decrease significantly from $10.2 \Omega$ to $2.09 \Omega$ when NiCoS is incorporated with HsGDY (NiCoS@HsGDY), suggesting much facilitated electron transfer by introducing HsGDY. A further decreased $R_{\text {ct }}$ value of NiCoS@HsGDY@Ni,Co-MoS $2(0.638 \Omega)$ clearly indicates the benefit of the triple-layer structure. When compared with $\mathrm{NiCoMoS}(0.748 \Omega)$, the lower $R_{\mathrm{ct}}$ value of NiCoS@HsGDY@Ni,Co-MoS $2(0.638 \Omega)$ also reveals the role of HsGDY as a built-in electron conductive channel during the HER process.

We further measure the specific surface area and the electrochemical active surface area (ECSA) of these nanoarray electrodes synthesized in this work. First, using cyclic voltammetry (CV) method, we determine the ECSA of NiCoS@HsGDY@Ni,Co- $\mathrm{MoS}_{2}$ and NiCoMoS by measuring the electrochemical double-layer capacitance $\left(C_{\mathrm{d}}\right)$, which is believed to be positively proportional to the $\mathrm{ECSA}^{73}$. As shown in the Supplementary Fig. 18, the $C_{\mathrm{dl}}$ of NiCoS@HsGDY@Ni,Co-MoS 2 $\left(75 \mathrm{mF} \mathrm{cm}^{-2}\right)$ is larger than that of NiCoMoS $\left(65.4 \mathrm{mF} \mathrm{cm}^{-2}\right)$, 

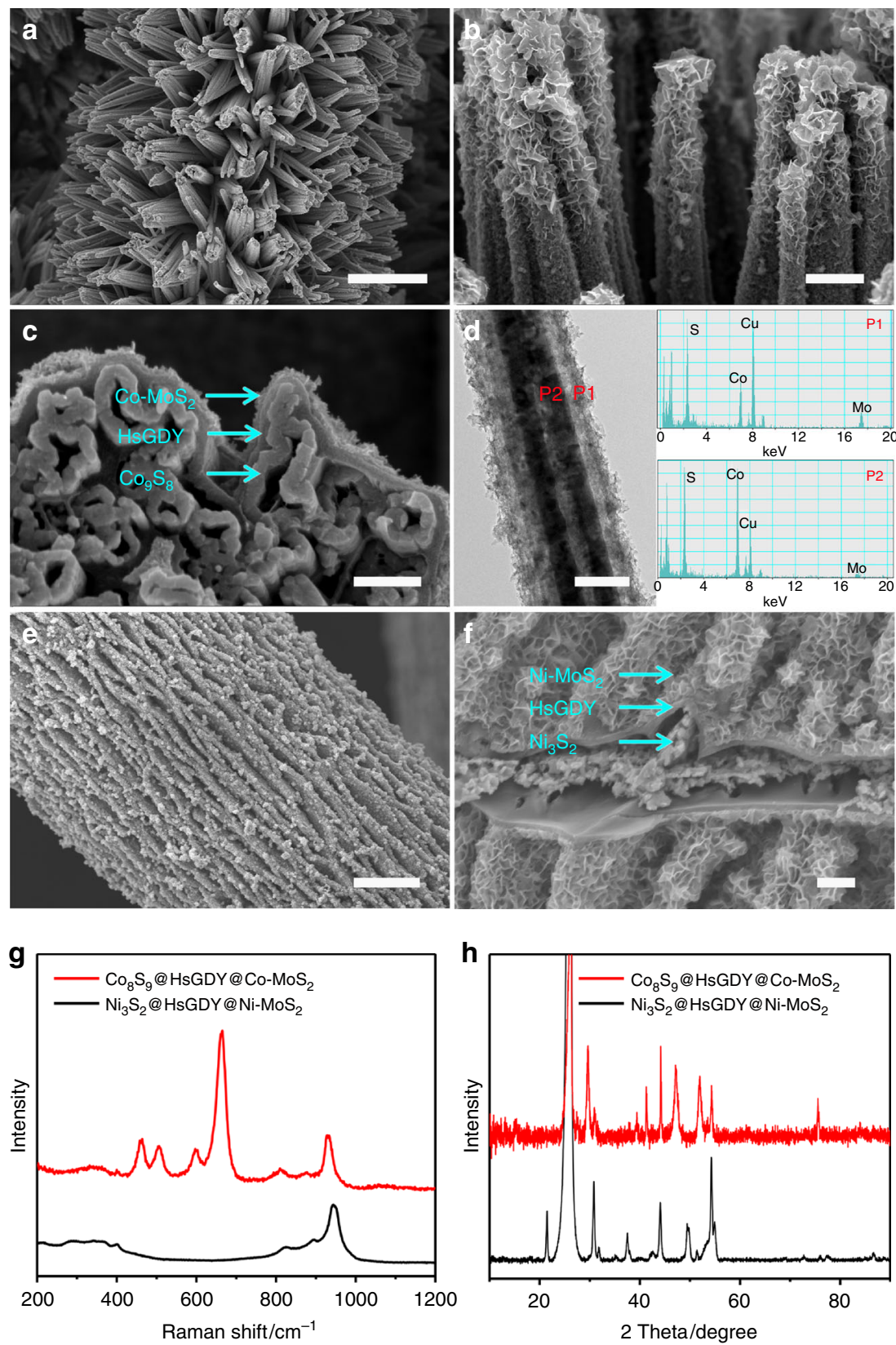

Fig. 6 Structural characterizations of tri-layered nanoarrays. a-c, e, $\mathbf{f}$ Scanning electron microscopy (SEM) images, $\mathbf{d}$ transmission electron microscopy (TEM) image with corresponding energy dispersive spectroscopy (EDS) spectra, $\mathbf{g}$ Raman spectra, and $\mathbf{h}$ X-ray diffraction (XRD) patterns of the tri-layered

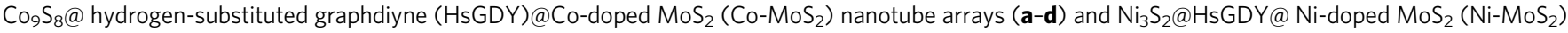
nanosheet arrays (e-f). Scale bars: a $5 \mu \mathrm{m}, \mathbf{b} 500 \mathrm{~nm}, \mathbf{c}, \mathbf{f} 200 \mathrm{~nm}, \mathbf{d} 100 \mathrm{~nm}$, e $2 \mu \mathrm{m}$

indicating that the tri-layered nanostructure allows better electrolyte/products transfer in and out through the entire electrode than that of mixed NiCoMoS. The BET-specific surface areas of NiCoS@HsGDY@Ni,Co-MoS, NiCoS@HsGDY, NiCo$\mathrm{MoS}, \mathrm{NiCoS}$, and carbon paper are $18.46,16.75,5.49,3.17$, and $0.75 \mathrm{~m}^{2} \mathrm{~g}^{-1}$, respectively (Supplementary Fig. 18), with the trilayered nanostructure shows the highest surface area. Notably, an inner-connective porous structure is obvious in the interconnected nanosheets of $\mathrm{Ni}, \mathrm{Co}-\mathrm{MoS}_{2}$, especially at the interface between $\mathrm{Ni}, \mathrm{Co}-\mathrm{MoS}_{2}$ and HsGDY (red circle in Supplementary Fig. 19). Besides, both NiCoS@HsGDY@Ni,Co-MoS 2 and HsGDY nanoarrays exhibit superhydrophilicity as shown in the Supplementary Fig. 20. Therefore, the electrolyte would permeate through the middle layer of microporous HsGDY, which acts as an ion channel that allows mass transfer through the micropores.
Interestingly, the inner layer of $\mathrm{NiCoS}$ with $\mathrm{HsGDY}$ coating is also porous as shown in the blue circle in the Supplementary Fig. 19, which further permits the electrolyte into the hollowedout cavity of the inner NiCoS layers. Therefore, the tri-layered nanoarrays in this work enable mass transfer through the entire electrode, which is desirable in HER and many other energyrelated applications.

It is confirmed that HsGDY has no HER activity when the overpotential is smaller than $300 \mathrm{mV}$, which, when combined with the enhanced HER performance of NiCoS@HsGDY@Ni,Co$\mathrm{MoS}_{2}$ against $\mathrm{NiCoMoS}$, verifies its HER inactivity and the function of a built-in electron and ion channel in the triplelayered structure. Thus, we conclude that the triple-layered tubular texture with a unique built-in electron and ion channel allows for efficient chemical reaction kinetics. 
a

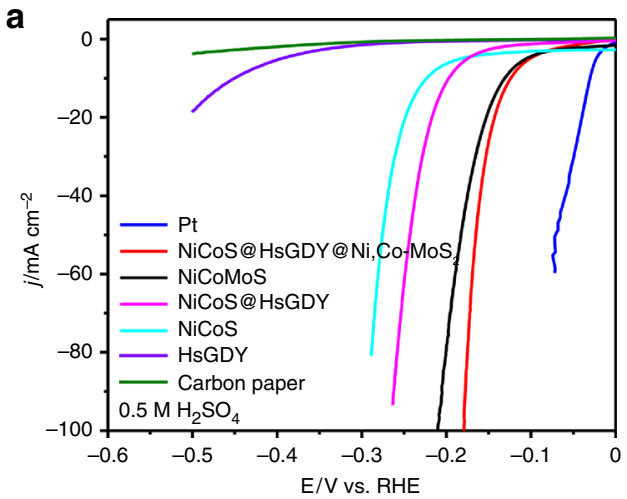

c

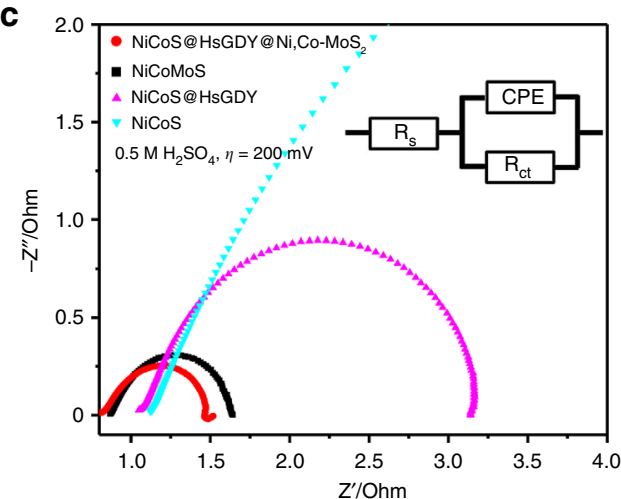

e

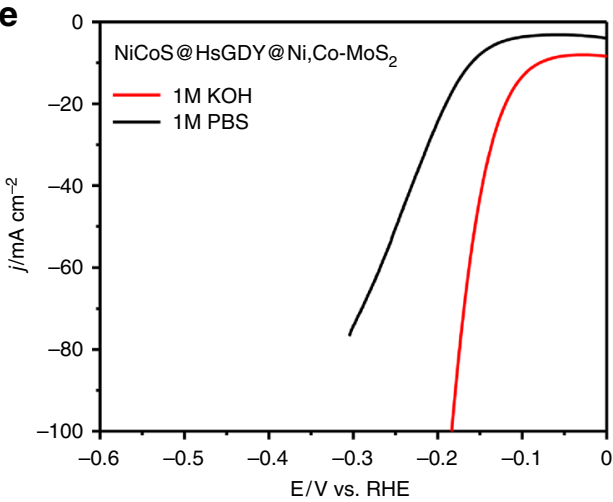

b

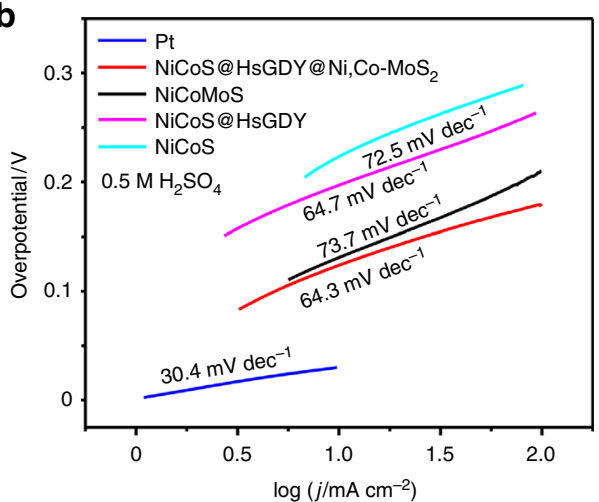

d

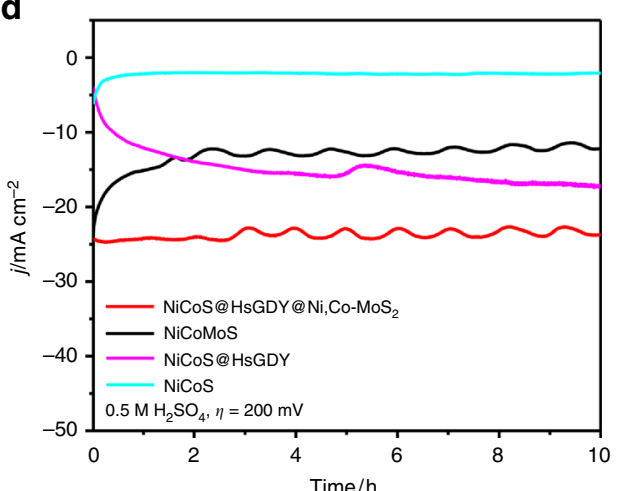

$f$

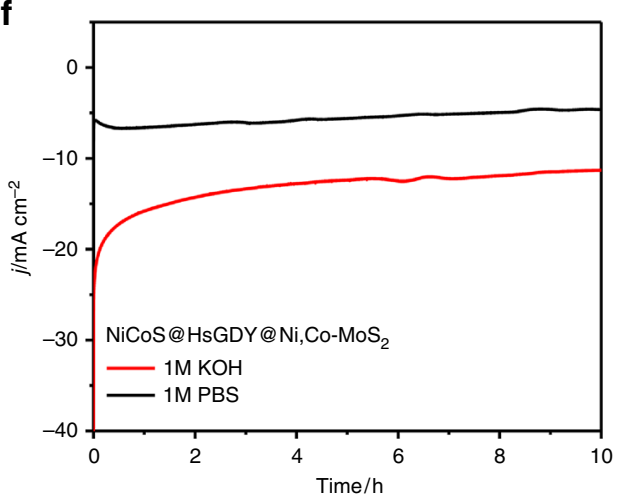

Fig. 7 Electrochemical evaluation of hydrogen evolution reaction performance. a Hydrogen evolution reaction (HER) polarization curves with iRcompensations, b Tafel plots, c Nyquist plots derived from electrochemical impedance spectroscopy (EIS), with an equivalent circuit (CPE constant phase element, $R_{\mathrm{s}}$ equivalent series resistance, $R_{\mathrm{ct}}$ charge transfer resistance), $\mathbf{d}$ time-dependent current density curves without $i R$-compensations of $\mathrm{Ni}_{3} \mathrm{~S}_{2} /$ $\mathrm{Co}_{9} \mathrm{~S}_{8}$ (NiCoS)@hydrogen-substituted graphdiyne (HsGDY)@Ni-,Co-co-doped $\mathrm{MoS}_{2}\left(\mathrm{Ni}, \mathrm{Co}-\mathrm{MoS}_{2}\right.$ ), mixed $\mathrm{Co}_{9} \mathrm{~S}_{8}, \mathrm{Ni}_{3} \mathrm{~S}_{2}$, and Ni,Co-MoS $2(\mathrm{NiCoMoS})$, NiCoS@HsGDY, NiCoS, HsGDY, Pt sheet and Carbon paper in $0.5 \mathrm{M} \mathrm{H}_{2} \mathrm{SO}_{4}(\mathrm{pH}=0.5)$. e HER polarization curves with iR-compensations and $\mathbf{f}$ timedependent current density curves without $i R$-compensations of $\mathrm{NiCoS} @ \mathrm{HsGDY} @ N i, C o-\mathrm{MoS}_{2}$ in $1 \mathrm{M} \mathrm{KOH}(\mathrm{pH}=14.1)$ and $1 \mathrm{M}$ phosphate buffer solution (PBS) $(\mathrm{pH}=7.1)$, respectively

Notably, a good HER performance with an overpotential of $100 \mathrm{mV} \mathrm{dec}^{-1}\left(10 \mathrm{~mA} \mathrm{~cm}^{-2}\right)$ and Tafel slop of $89.5 \mathrm{mV} \mathrm{dec}^{-1}$ is achieved with tri-layered NiCoS@HsGDY@Ni,Co-MoS 2 , when performed in the alkaline solution $(1 \mathrm{M} \mathrm{KOH})$ (Fig. 7e and Supplementary Fig. 21). An overpotential of $160 \mathrm{mV}$ at $10 \mathrm{~mA}$ $\mathrm{cm}^{-1}$ is also acquired in $1 \mathrm{M}$ phosphate buffer solution (PBS) $(\mathrm{pH}=7.1)$ (Fig. $7 \mathrm{e}$ and Supplementary Fig. 21). It has been reported that metal chalcogenides (e.g., $\mathrm{MoS}_{2}$ ) doped with secondary metal ions (e.g., $\mathrm{Co}, \mathrm{Ni}$ ) could be good candidates to perform HER in alkaline and neutral media with enhanced activity and stability ${ }^{57,74}$. The incorporation of heteroatoms is considered to promote the initial discharge of water to form the hydrogen intermediates $\left(\mathrm{H}_{\mathrm{ad}}\right)$ for HER in alkaline and neutral media, which consequently facilitates the generation of $\mathrm{H}_{2}$. Therefore, the incorporation of $\mathrm{Ni}$ and Co into $\mathrm{MoS}_{2}$ leads to the enhanced HER performance within a wide $\mathrm{pH}$ range. Besides, the stability of the core structure of $\mathrm{Ni}_{3} \mathrm{~S}_{2} / \mathrm{Co}_{9} \mathrm{~S}_{8}$ is also considerably promoted as a result of the seamlessly coated HsGDY layers.

As shown in Fig. 7d, f, the tri-layered NiCoS@HsGDY@Ni,Co$\mathrm{MoS}_{2}$ nanoarrays exhibit stability with $96 \%$ retention of the initial current in $0.5 \mathrm{M} \mathrm{H}_{2} \mathrm{SO}_{4}(\mathrm{pH}=0.5)$ and $80 \%$ performance retention in $1 \mathrm{M}$ PBS $(\mathrm{pH}=7.1)$ after $10 \mathrm{~h}$ of HER. With $1 \mathrm{M}$ $\mathrm{KOH}(\mathrm{pH}=14.1)$, there is an initial current-density decrease within the first $30 \mathrm{~min}$, but a stable current persists afterward for $10 \mathrm{~h}$ of the HER. The SEM images show that the morphology of 
the tri-layered NiCoS@HsGDY@Ni,Co- $\mathrm{MoS}_{2}$ nanoarrays is well preserved under three $\mathrm{pH}$ conditions for $10 \mathrm{~h}$ of HER (Supplementary Fig. 22). It should be noted that although the middle $\mathrm{HsGDY}$ layers could effectively protect the core of $\mathrm{Ni}_{3} \mathrm{~S}_{2} /$ $\mathrm{Co}_{9} \mathrm{~S}_{8}$ in alkaline electrolyte, a degree of reduction in mass transfer capability still occurred in the initial stages caused by the partial dissolution/aggregation of $\mathrm{Ni}_{3} \mathrm{~S}_{2} / \mathrm{Co}_{9} \mathrm{~S}_{8}$ (Supplementary Fig. 23). However, the built-in HsGDY layers lead to the longterm stability of the tri-layered NiCoS@HsGDY@Ni,Co-MoS nanoarrays beyond the initial stage. These results unequivocally reveal the stability of the tri-layered NiCoS@HsGDY@Ni,Co$\mathrm{MoS}_{2}$ nanoarrays in this work. We further compare the HER stability of NiCoS@HsGDY@Ni,Co- $\mathrm{MoS}_{2}, \quad \mathrm{NiCoMoS}$, NiCoS@HsGDY and NiCoS for a duration of $10 \mathrm{~h}$ in $0.5 \mathrm{M}$ $\mathrm{H}_{2} \mathrm{SO}_{4}(\mathrm{pH}=0.5)$. As shown in Fig. $7 \mathrm{~d}$, the well-aligned singlelayered NiCoS nanoarrays are completely destroyed after $10 \mathrm{~h}$ HER process. However, the activity and stability of the core structure of $\mathrm{Ni}_{3} \mathrm{~S}_{2} / \mathrm{Co}_{9} \mathrm{~S}_{8}$ is considerably promoted in the presence of the HsGDY coating layer during HER (Supplementary Fig. 22). Besides, the HER performance of NiCoS@HsGDY@Ni,Co-MoS 2 against $\mathrm{NiCoMoS}$ indicates the contribution of the core structure of NiCoS@HsGDY, which is otherwise destroyed without HsGDY protection after $2 \mathrm{~h}$ of HER (Fig. $7 \mathrm{~d}$ ). These results reveal the benefits of the built-in HsGDY layers to the HER performance of the electrode in both activity and stability.

The HER performance of NiCoS@HsGDY@Ni,Co- $\mathrm{MoS}_{2}$ is better than many of reported materials of $2 \mathrm{H}-\mathrm{MoS}_{2}, \mathrm{Co}_{9} \mathrm{~S}_{8}, \mathrm{Ni}_{3} \mathrm{~S}_{2}$ (Supplementary Table 1). The effective HER performance of NiCoS@HsGDY@Ni,Co- $\mathrm{MoS}_{2}$ in a wide range of pH values is attributed to the following factors. First, the incorporation of $\mathrm{Ni}$ and Co in the Mo-S phase in the inner layer leads to multimetallic sulfides with rich defects; secondly, the seamless sandwiched conductive HsGDY middle layer as a built-in electron and ion channel improves electrode kinetics owing to the large extended $\pi$-conjugated system; thirdly, the triple-layered tubular and edge-terminated texture and unique hierarchical porous nanostructure allows for exposure of more active sites and easy electrolyte permeation. Fourthly, the seamless and intimate contact between the HsGDY and both the inner and outer layers effectively prevent them from aggregation during the HER process. All of the above factors contribute to the excellent performance and durability of NiCoS@HsGDY@Ni,Co-MoS which shows well-preserved structure and performance after $10 \mathrm{~h}$ of HER evaluation. Therefore, we believe that these triple-layered nanostructures of HsGDY-sandwiched TMCs would be promising in other electrochemical applications, such as supercapacitors $^{75}, \mathrm{Na} / \mathrm{Li}$ ion storage ${ }^{50}$, oxygen evolution reaction ${ }^{76}, \mathrm{CO}_{2}$ reduction ${ }^{77}$, and water desalination ${ }^{78}$ etc.

\section{Discussion}

In conclusion, this work reports a dual-template strategy that involves using HsGDY, one type of MONs, to coat a self-template of transition metals. Under suitable chemical reaction conditions, the dual template can lead to fabrication of triple-layered nanostructures in which each layer has a different chemical composition and nanostructure. Inert HsGDY is judiciously selected for this synthesis, as it physically confines the sacrificial template in place and simultaneously allows mass transfer through its micropores. Based on a dual-template of $\mathrm{Ni}-\mathrm{Co}$ hydroxyl-carbonate nanoarays confined with a conformally coated HsGDY layer, triple-layered nanotube arrays composed of a HsGDY middle layer sandwiched between an external layer of Ni-,Co-co-doped $\mathrm{MoS}_{2}$ nanosheets and an internal layer of NiCoS $\left(\mathrm{Co}_{9} \mathrm{~S}_{8}, \mathrm{Ni}_{3} \mathrm{~S}_{2}\right)$ nanotubular layer has been elaborately fabricated. The synthesis is versatile and grows the triple-layered nanostructures directly on conductive carbon papers, which makes readily it a suitable electrode material for many electrochemical applications. The triple-layered material delivers a HER performance better than most of the corresponding $2 \mathrm{H}-\mathrm{MoS}_{2}$, $\mathrm{Ni}_{3} \mathrm{~S}_{2}$ and $\mathrm{Co}_{9} \mathrm{~S}_{8}$ over a wide range of $\mathrm{pH}$ values $(0-14)$. This work contributes to the ongoing search for multi-layered nanostructures towards advanced energy conversion/storage applications.

\section{Methods}

Synthesis of $(\mathrm{Ni}, \mathrm{Co})(\mathrm{OH})_{2} \mathrm{CO}_{3}(\mathrm{NiCoHC})$ nanowire arrays. Ni-Co hydroxylcarbonate nanowire arrays were directly grown on the carbon fibre paper according to a literature method ${ }^{32}$. Briefly, $0.95 \mathrm{~g}$ of $\mathrm{CoCl}_{2} \cdot 6 \mathrm{H}_{2} \mathrm{O}, 0.475 \mathrm{~g}$ of $\mathrm{NiCl}_{2} \cdot 6 \mathrm{H}_{2} \mathrm{O}$, $0.43 \mathrm{~g}$ of urea, and $0.58 \mathrm{~g}$ of cetyltrimethylammonium bromide (CTAB) were dissolved into $40 \mathrm{~mL}$ of deionized water under sonication to form a clear pink solution. Then, the solution was transferred to a $50 \mathrm{~mL}$ Teflon-lined stainless steel autoclave containing the precleaned carbon paper (FuelCellsEtc, AvCarb MGL370, $2.5 \times 6 \mathrm{~cm}^{2}$, see Supplementary Information for details). After hydrothermal treatment at $100{ }^{\circ} \mathrm{C}$ for $10 \mathrm{~h}$ in an oven, the carbon paper with $\mathrm{NiCoHC}$ nanowire arrays was taken out of the autoclave, and then cleaned by ultrasonication with DI water and ethanol repeatedly to remove the loosely attached products.

Synthesis of core-shell nanowire arrays. A conformal layer of HsGDY was directly coated on the surface of the $\mathrm{NiCoHC}$ nanowire arrays. In details, the carbon paper with $\mathrm{NiCoHC}$ nanowire arrays on its surface $\left(1 \times 6 \mathrm{~cm}^{2}\right)$ was fixed in a $100 \mathrm{~mL}$ pear-shaped flask containing tetrahydrofuran $(20 \mathrm{~mL})$ and trimethylamine $(40 \mathrm{~mL})$ with catalysts of $\mathrm{Pd}\left(\mathrm{PPh}_{3}\right)_{2} \mathrm{Cl}_{2}(16.8 \mathrm{mg})$ and $\mathrm{CuI}(4.4 \mathrm{mg})$. After stirring at room temperature for 30 mins under argon atmosphere, 1,3,5-triethynylbenzene $(10 \mathrm{mg})$ was added. Then the reactor was sealed and heated at $60{ }^{\circ} \mathrm{C}$ for $24 \mathrm{~h}$ under argon atmosphere. Microporous HsGDY networks gradually generated on the surface of the nanowire arrays through Glaser coupling of 1,3,5-triethynylbenzene. The conformal NiCoHC@HsGDY nanowire arrays with a color of brown was obtained by ultrasonication with ethanol.

Synthesis of tri-layered nanotube arrays. Then, the as-prepared NiCoHC@HsGDY nanoarrays were utilized as a dual template to construct triplelayered nanostructures by the reaction with $\left(\mathrm{NH}_{4}\right)_{2} \mathrm{MoS}_{4}$ in the presence of $\mathrm{N}_{2} \mathrm{H}_{4}$ In details, the carbon paper with the surface NiCoHC@HsGDY nanowire arrays $\left(1 \times 2 \mathrm{~cm}^{2}\right)$ was placed in an autoclave containing $10 \mathrm{~mL} \mathrm{DMF}, 10 \mathrm{mg}$

$\left(\mathrm{NH}_{4}\right)_{2} \mathrm{MoS}_{4}$, and $30 \mu \mathrm{L} \mathrm{N} \mathrm{N}_{2} \mathrm{H}_{4}$ under the protection of Argon gas. The autoclave was subjected to $15 \mathrm{~h} 210{ }^{\circ} \mathrm{C}$ hydrothermal process, by the end of which the triplelayered NiCoS@HsGDY@Ni,Co- $\mathrm{MoS}_{2}$ nanotube arrays with a color of black was acquired. The carbon paper was then sonicated with water and ethanol to remove the loosely attached products. For the purpose of confirmation, triple-layered nanoarrays of $\mathrm{Co}_{9} \mathrm{~S}_{8} @ \mathrm{HsGDY} @ \mathrm{Co}-\mathrm{MoS}_{2}$, and nanosheet arrays of $\mathrm{Ni}_{3} \mathrm{~S}_{2} @ \mathrm{HsG}$ DY@Ni- $\mathrm{MoS}_{2}$ were also synthesized when $\mathrm{Co}(\mathrm{OH}) \mathrm{F} @ \mathrm{HsGDY}$ nanowire arrays and $\mathrm{Ni}(\mathrm{OH}) \mathrm{F} @ \mathrm{HsGDY}$ nanosheet arrays were used as dual-template with other conditions being all the same (see Supplementary Information). NiCoS and NiCoS@HsGDY nanoarrays were further prepared purposefully to verify the HER performance of the core structures and reveal the function of built-in HsGDY layers (see Supplementary Information).

Characterization. The SEM images were taken with a Zeiss Merlin scanning electron microscope. TEM, high-resolution TEM (HRTEM), HAADF-STEM images, EELS, and STEM-EELS elemental mapping were obtained with a ThermoFisher Scientific's Titan ST equipped with a Gatan Image Filter (GIF) Tridiem. The ultrathin sections $(70 \mathrm{~nm})$ cured by epoxy resin were prepared on an Ultramicrotome with a glass blade and then placed on a carbon-coated copper grid followed by TEM imaging. The XRD patterns of the products were recorded with Bruker D8 ADVANCE Diffraction System using a Cu Ka source $(\lambda=1.5406 \AA)$. Raman spectra was collected on a Horiba Aramis with a $473 \mathrm{~nm}$ laser wavelength excitation and spectra were up to $3000 \mathrm{~cm}^{-1}$. XPS was carried out on an Axis Ultra instrument (Kratos Analytical) under ultrahigh vacuum $\left(<10^{-9} \mathrm{mbar}\right)$ by using a monochromatic Al Ka X-ray source $(\mathrm{hv}=1486.6 \mathrm{eV})$ operated at $150 \mathrm{~W}$. Solid NMR spectra were acquired using WB Bruker 600 AVANAC III spectrometer equipped with $2.5 \mathrm{~mm}$ double resonance MAS Bruker Probe (BrukerBioSpin, Rheinstetten, Germany). The surface area and pore size distributions of the synthesized materials were determined by a Micromeeitics-TriStar II system. The surface area was calculated using the BET method and the pore size distributions were calculated using the Density Functional Theory (DFT) from the desorption branch. The Fourier transform infrared spectroscopy (FTIR) was recorded on a FTIR-is10 spectrometer using a diamond. See Supporting Information for the details of XPS and solid NMR. Static contact angles were conducted with a commercial contact angle system (OCA 35, DataPhysics, Filderstadt, Germany) to quantify the hydrophilicity of the synthesized nanostructures. 
Electrocatalytic HER. Electrochemical measurements were carried out in a threeelectrode system consisting of a working electrode, a graphit rod counter electrode, and an $\mathrm{Ag} / \mathrm{AgCl}(3 \mathrm{M} \mathrm{KCl})$ reference electrode conducted with Autolab PGSTAT $302 \mathrm{~N}$ potentiostat (Metrohm Autolab, Netherlands). All the measured potentials were referred to reverse hydrogen electrode (RHE) with the equation of $E(\mathrm{RHE})=$ $E\left(\mathrm{Ag}^{+} / \mathrm{AgCl}\right)+0.210 \mathrm{~V}+0.0591 \mathrm{pH}$. The aqueous solution of $0.5 \mathrm{M} \mathrm{H}_{2} \mathrm{SO}_{4}(\mathrm{pH}$ $=0.5), 1 \mathrm{M}$ phosphate buffer solution ( $\mathrm{PBS}, \mathrm{pH}=7.1)$ and $1 \mathrm{M} \mathrm{KOH}(\mathrm{pH}=14.1)$ were utilized to evaluate their HER performance over a wide $\mathrm{pH}$ values. The linear sweep voltammetry (LSV) for water reduction was carried out at a scan rate of $5 \mathrm{mV} \mathrm{s}^{-1}$. The polarization curves were plotted as overpotential $(\eta)$ verse log current $(\log j)$ which was used to evaluate the Tafel slopes by fitting the linear portion to the Tafel equation $(\eta=\mathrm{b} \log j+\mathrm{a})$. All polarization curves were $i R$ corrected. The EIS was performed at the overpotential of $200 \mathrm{mV}$ from $100 \mathrm{kHz}$ to $10 \mathrm{mHz}$. The time-dependent current density (I-t) curves were measured at overpotential of $200 \mathrm{mV}$.

Data availability. The data that support the findings of this study are available from the corresponding authors on request.

Received: 18 January 2018 Accepted: 10 July 2018

Published online: 07 August 2018

\section{References}

1. Yu, L., Hu, H., Wu, H. B. \& Lou, X. W. D. Complex hollow nanostructures: synthesis and energy-related applications. Adv. Mater. 29, 1604563 (2017).

2. Prieto, G. et al. Hollow nano- and microstructures as catalysts. Chem. Rev. 116, 14056-14119 (2016)

3. Wang, X., Feng, J., Bai, Y., Zhang, Q. \& Yin, Y. Synthesis, properties, and applications of hollow micro-/nanostructures. Chem. Rev. 116, 10983-11060 (2016).

4. Qi, J. et al. Multi-shelled hollow micro-/nanostructures. Chem. Soc. Rev. 44, 6749-6773 (2015).

5. Becknell, N. et al. Control of architecture in rhombic dodecahedral Pt-Ni nanoframe electrocatalysts. J. Am. Chem. Soc. 139, 11678-11681 (2017).

6. Liu, W. et al. Multi-shelled hollow metal-organic frameworks. Angew. Chem. Int. Ed. 56, 5512-5516 (2017).

7. Bin, D.-S. et al. Controlling the compositional chemistry in single nanoparticles for functional hollow carbon nanospheres. J. Am. Chem. Soc. 139, 13492-13498 (2017).

8. Yang, Y. et al. Multi-shelled dendritic mesoporous organosilica hollow spheres: roles of composition and architecture in cancer immunotherapy. Angew. Chem. Int. Ed. 56, 8446-8450 (2017).

9. Wang, J. et al. Multi-shelled metal oxides prepared via an anion-adsorption mechanism for lithium-ion batteries. Nat. Energy 1, 16050 (2016).

10. Shen, L. et al. Formation of nickel cobalt sulfide ball-in-ball hollow spheres with enhanced electrochemical pseudocapacitive properties. Nat. Commun. 6 , 6694 (2015).

11. Hu, H., Guan, B. Y. \& Lou, X. W. D. Construction of complex CoS hollow structures with enhanced electrochemical properties for hybrid supercapacitors. Chem 1, 102-113 (2016).

12. Sun, $\mathrm{H}$. et al. Porous multishelled $\mathrm{Ni}_{2} \mathrm{P}$ hollow microspheres as an active electrocatalyst for hydrogen and oxygen evolution. Chem. Mater. 29, 8539-8547 (2017).

13. Wang, J. et al. Accurate control of multishelled $\mathrm{Co}_{3} \mathrm{O}_{4}$ hollow microspheres as high-performance anode materials in lithium-ion batteries. Angew. Chem. Int. Ed. 52, 6417-6420 (2013).

14. Zhao, X. et al. Formation of septuple-shelled $\left(\mathrm{Co}_{2 / 3} \mathrm{Mn}_{1 / 3}\right)\left(\mathrm{Co}_{5 / 6} \mathrm{Mn}_{1 / 6}\right)_{2} \mathrm{O}_{4}$ hollow spheres as electrode material for alkaline rechargeable battery. $A d v$. Mater. 29, 1700550 (2017).

15. Zhang, J., Hu, H., Li, Z. \& Lou, X. W. D. Double-shelled nanocages with cobalt hydroxide inner shell and layered double hydroxides outer shell as highefficiency polysulfide mediator for lithium-sulfur batteries. Angew. Chem. Int. Ed. 55, 3982-3986 (2016).

16. Hu, H., Guan, B., Xia, B. \& Lou, X. W. D. Designed formation of $\mathrm{Co}_{3} \mathrm{O}_{4} /$ $\mathrm{NiCO}_{2} \mathrm{O}_{4}$ double-shelled nanocages with enhanced pseudocapacitive and electrocatalytic properties. J. Am. Chem. Soc. 137, 5590-5595 (2015).

17. Zhang, G. et al. General formation of complex tubular nanostructures of metal oxides for the oxygen reduction reaction and lithium-ion batteries. Angew. Chem. Int. Ed. 52, 8643-8647 (2013).

18. Zhao, M. et al. Metal-organic frameworks as selectivity regulators for hydrogenation reactions. Nature 539, 76-80 (2016).

19. Zhan, W.-W. et al. Semiconductor@metal-organic framework core-shell heterostructures: a case of ZnO@ZIF-8 nanorods with selective photoelectrochemical response. J. Am. Chem. Soc. 135, 1926-1933 (2013).
20. Lu, S. et al. Synthesis of ultrafine and highly dispersed metal nanoparticles confined in a thioether-containing covalent organic framework and their catalytic applications. J. Am. Chem. Soc. 139, 17082-17088 (2017).

21. Li, H. et al. Amorphous nickel-cobalt complexes hybridized with 1T-phase molybdenum disulfide via hydrazine-induced phase transformation for water splitting. Nat. Commun. 8, 15377 (2017).

22. Shang, H. et al. Ultrathin graphdiyne nanosheets grown in situ on copper nanowires and their performance as lithium-ion battery anodes. Angew. Chem. Int. Ed. 57, 774-778 (2018).

23. Su, H. et al. Activating cobalt nanoparticles via the Mott-Schottky effect in nitrogen-rich carbon shells for base-free aerobic oxidation of alcohols to esters. J. Am. Chem. Soc. 139, 811-818 (2017).

24. Xu, Y. et al. Nickel nanoparticles encapsulated in few-layer nitrogen-doped graphene derived from metal-organic frameworks as efficient bifunctional electrocatalysts for overall water splitting. Adv. Mater. 29, 1605957 (2017).

25. Wang, S., Guan, B. Y., Yu, L. \& Lou, X. W. D. Rational design of three-layered $\mathrm{TiO}_{2} @$ carbon@ $@ \mathrm{MoS}_{2}$ hierarchical nanotubes for enhanced lithium storage. Adv. Mater. 29, 1702724 (2017)

26. Chen, T. et al. Self-templated formation of interlaced carbon nanotubes threaded hollow $\mathrm{Co}_{3} \mathrm{~S}_{4}$ nanoboxes for high-rate and heat-resistant lithiumsulfur batteries. J. Am. Chem. Soc. 139, 12710-12715 (2017).

27. Dong, $\mathrm{Y}$. et al. Air-stable porous $\mathrm{Fe}_{2} \mathrm{~N}$ encapsulated in carbon microboxes with high volumetric lithium storage capacity and a long cycle life. Nano. Lett. 17, 5740-5746 (2017).

28. Wei, Q. et al. Porous one-dimensional nanomaterials: design, fabrication and applications in electrochemical energy storage. Adv. Mater. 29, 1602300 (2017).

29. Zhang, H., Huang, X., Noonan, O., Zhou, L. \& Yu, C. Tailored yolk-shell Sn@C nanoboxes for high-performance lithium storage. Adv. Funct. Mater. 27, 1606023 (2017)

30. Yang, L.-P. et al. General synthetic strategy for hollow hybrid microspheres through a progressive inward crystallization process. J. Am. Chem. Soc. 138, 5916-5922 (2016)

31. Cai, G., Zhang, W., Jiao, L., Yu, S.-H. \& Jiang, H.-L. Template-directed growth of well-aligned MOF arrays and derived self-supporting electrodes for water splitting. Chem 2, 791-802 (2017)

32. Bae, S.-H. et al. Seamlessly conductive 3D nanoarchitecture of core-shell NiCo nanowire network for highly efficient oxygen evolution. Adv. Energy Mater. 7, 1601492 (2017)

33. Wang, J., Zhong, H.-X., Wang, Z.-L., Meng, F.-L. \& Zhang, X.-B. Integrated three-dimensional carbon paper/carbon tubes/cobalt-sulfide sheets as an efficient electrode for overall water splitting. ACS Nano 10, 2342-2348 (2016).

34. Ma, T. Y., Dai, S., Jaroniec, M. \& Qiao, S. Z. Metal-organic framework derived hybrid $\mathrm{Co}_{3} \mathrm{O}_{4}$-carbon porous nanowire arrays as reversible oxygen evolution electrodes. J. Am. Chem. Soc. 136, 13925-13931 (2014).

35. Deng, S. et al. Hollow $\mathrm{TiO}_{2} @ \mathrm{Co}_{9} \mathrm{~S}_{8}$ core-branch arrays as bifunctional electrocatalysts for efficient oxygen/hydrogen production. Adv. Sci. 5, 1700772 (2017).

36. Zhang, J. et al. Interface engineering of $\mathrm{MoS}_{2} / \mathrm{Ni}_{3} \mathrm{~S}_{2}$ heterostructures for highly enhanced electrochemical overall-water-splitting activity. Angew. Chem. Int. Ed. 55, 6702-6707 (2016).

37. Xiao, J., Wan, L., Yang, S., Xiao, F. \& Wang, S. Design hierarchical electrodes with highly conductive $\mathrm{NiCo}_{2} \mathrm{~S}_{4}$ nanotube arrays grown on carbon fibre paper for high-performance pseudocapacitors. Nano. Lett. 14, 831-838 (2014).

38. Liu, J., Zhu, D., Guo, C., Vasileff, A. \& Qiao, S.-Z. Design strategies toward advanced MOF-derived electrocatalysts for energy-conversion reactions. $A d v$. Energy Mater. 7, 1700518 (2017).

39. Huang, Z.-F. et al. Hollow cobalt-based bimetallic sulfide polyhedra for efficient all-pH-value electrochemical and photocatalytic hydrogen evolution. J. Am. Chem. Soc. 138, 1359-1365 (2016).

40. Zhao, W., Zhang, C., Geng, F., Zhuo, S. \& Zhang, B. Nanoporous hollow transition metal chalcogenide nanosheets synthesized via the anion-exchange reaction of metal hydroxides with chalcogenide ions. ACS Nano $\mathbf{8}$, 10909-10919 (2014)

41. Zhuo, S., Xu, Y., Zhao, W., Zhang, J. \& Zhang, B. Hierarchical nanosheetbased $\mathrm{MoS}_{2}$ nanotubes fabricated by an anion-exchange reaction of $\mathrm{MoO}_{3}$-amine hybrid nanowires. Angew. Chem. Int. Ed. 52, 8602-8606 (2013).

42. Liu, Y., Goebl, J. \& Yin, Y. Templated synthesis of nanostructured materials. Chem. Soc. Rev. 42, 2610-2653 (2013).

43. Liang, H.-W., Liu, S. \& Yu, S.-H. Controlled synthesis of one-dimensional inorganic nanostructures using pre-existing one-dimensional nanostructures as templates. Adv. Mater. 22, 3925-3937 (2010).

44. Jin, E. et al. Two-dimensional $\mathrm{sp}^{2}$ carbon-conjugated covalent organic frameworks. Science 357, 673-676 (2017)

45. Chun, J. et al. Metal-organic framework@microporous organic network: hydrophobic adsorbents with a crystalline inner porosity. J. Am. Chem. Soc. 136, 6786-6789 (2014). 
46. Xu, Y., Jin, S., Xu, H., Nagai, A. \& Jiang, D. Conjugated microporous polymers: design, synthesis and application. Chem. Soc. Rev. 42, 8012-8031 (2013).

47. Ding, S.-Y. \& Wang, W. Covalent organic frameworks (COFs): from design to applications. Chem. Soc. Rev. 42, 548-568 (2013).

48. Kang, N. et al. Microporous organic network hollow spheres: useful templates for nanoparticulate $\mathrm{Co}_{3} \mathrm{O}_{4}$ hollow oxidation catalysts. J. Am. Chem. Soc. 135, 19115-19118 (2013).

49. $\mathrm{Wu}, \mathrm{D}$. et al. Design and preparation of porous polymers. Chem. Rev. 112, 3959-4015 (2012).

50. He, J. et al. Hydrogen substituted graphdiyne as carbon-rich flexible electrode for lithium and sodium ion batteries. Nat. Commun. 8, 1172 (2017).

51. Wang, Z. et al. Water-dispersible hollow microporous organic network spheres as substrate for electroless deposition of ultrafine Pd nanoparticles with high catalytic activity and recyclability. Chem. Asian J. 11, 3178-3182 (2016).

52. Hu, Y., Liu, Y., Wang, Z. \& Zhang, B. Spontaneous electroless deposition of ultrafine Pd nanoparticles on poly(phenylene butadiynylene)s for the hydroxycarbonylation of aryl iodides. ChemistrySelect 1, 1832-1836 (2016).

53. Zhang, T. et al. Copper-surface-mediated synthesis of acetylenic carbon-rich nanofibres for active metal-free photocathodes. Nat. Commun. 9, 1140 (2018).

54. Zou, X. \& Zhang, Y. Noble metal-free hydrogen evolution catalysts for water splitting. Chem. Soc. Rev. 44, 5148-5180 (2015).

55. Seh, Z. W. et al. Combining theory and experiment in electrocatalysis: insights into materials design. Science 355, eaad4998 (2017).

56. Shi, Y. et al. Energy level engineering of $\mathrm{MoS}_{2}$ by transition-metal doping for accelerating hydrogen evolution reaction. J. Am. Chem. Soc. 139, 15479-15485 (2017).

57. Miao, J. et al. Hierarchical Ni-Mo-S nanosheets on carbon fibre cloth: a flexible electrode for efficient hydrogen generation in neutral electrolyte. Sci. Adv. 1, e1500259 (2015).

58. Merki, D., Vrubel, H., Rovelli, L., Fierro, S. \& Hu, X. Fe, Co, and Ni ions promote the catalytic activity of amorphous molybdenum sulfide films for hydrogen evolution. Chem. Sci. 3, 2515-2525 (2012).

59. Jiang, J., Gao, M., Sheng, W. \& Yan, Y. Hollow chevrel-phase $\mathrm{NiMo}_{3} \mathrm{~S}_{4}$ for hydrogen evolution in alkaline electrolytes. Angew. Chem. Int. Ed. 55, 15240-15245 (2016)

60. Deng, J. et al. Triggering the electrocatalytic hydrogen evolution activity of the inert two-dimensional $\mathrm{MoS}_{2}$ surface via single-atom metal doping. Energy Environ. Sci. 8, 1594-1601 (2015).

61. Deng, J. et al. Multiscale structural and electronic control of molybdenum disulfide foam for highly efficient hydrogen production. Nat. Commun. 8, 14430 (2017).

62. Yang, L. et al. Hierarchical spheres constructed by defect-rich $\mathrm{MoS}_{2} /$ carbon nanosheets for efficient electrocatalytic hydrogen evolution. Nano Energy 22, 490-498 (2016).

63. $\mathrm{Li}, \mathrm{Y}$. et al. $\mathrm{MoS}_{2}$ nanoparticles grown on graphene: an advanced catalyst for the hydrogen evolution reaction. J. Am. Chem. Soc. 133, 7296-7299 (2011)

64. Cao, J., Zhou, J., Zhang, Y., Wang, Y., \& Liu, X. Dominating role of aligned $\mathrm{MoS}_{2} / \mathrm{Ni}_{3} \mathrm{~S}_{2}$ nanoarrays supported on three-dimensional $\mathrm{Ni}$ foam with hydrophilic interface for highly enhanced hydrogen evolution reaction. ACS Appl. Mater. Interfaces 10, 1752-1760 (2018).

65. Zhu, H. et al. When cubic cobalt sulfide meets layered molybdenum disulfide: a core-shell system toward synergetic electrocatalytic water splitting. $A d v$. Mater. 27, 4752-4759 (2015).

66. Jiang, J.-X. et al. Conjugated microporous poly(phenylene butadiynylene)s. Chem. Commun. 486-488 (2008)

67. Xue, Y. et al. Self-catalyzed growth of Cu@graphdiyne core-shell nanowires array for high efficient hydrogen evolution cathode. Nano Energy 30, 858-866 (2016).

68. Feng, J.-X., Wu, J.-Q., Tong, Y. \& Li, G.-R. Efficient hydrogen evolution on Cu nanodots-decorated $\mathrm{Ni}_{3} \mathrm{~S}_{2}$ nanotubes by optimizing atomic hydrogen adsorption and desorption. J. Am. Chem. Soc. 140, 610-617 (2018).

69. Xiong, D. et al. Vertically aligned $\mathrm{Co}_{9} \mathrm{~S}_{8}$ nanotube arrays onto graphene papers as high-performance flexible electrodes for supercapacitors. Chem. Eur. J. 24, 2339-2343 (2017).
70. Sarno, M. \& Ponticorvo, E. Effect of the amount of nickel sulphide, molybdenum disulphide and carbon nanosupport on a tafel slope and overpotential optimization. Nanotechnology 28, 214003 (2017).

71. Zhou, W. et al. $\mathrm{Ni}_{3} \mathrm{~S}_{2}$ nanorods/Ni foam composite electrode with low overpotential for electrocatalytic oxygen evolution. Energy Environ. Sci. 6 , 2921-2924 (2013).

72. Han, X. et al. Hydrogen evolution reaction on hybrid catalysts of vertical $\mathrm{MoS}_{2}$ nanosheets and hydrogenated graphene. ACS Catal. 8, 1828-1836 (2018).

73. Chen, Y.-Y. et al. Pomegranate-like N,P-doped $\mathrm{Mo}_{2} \mathrm{C} @ \mathrm{C}$ nanospheres as highly active electrocatalysts for alkaline hydrogen evolution. ACS Nano 10, 8851-8860 (2016).

74. Staszak-Jirkovsky, J. et al. Design of active and stable Co-Mo-S $\mathrm{S}_{\mathrm{x}}$ chalcogels as $\mathrm{pH}$-universal catalysts for the hydrogen evolution reaction. Nat. Mater. 15, 197-203 (2016).

75. Yun, Q., Lu, Q., Zhang, X., Tan, C. \& Zhang, H. Three-dimensional architectures constructed from transition-metal dichalcogenide nanomaterials for electrochemical energy storage and conversion. Angew. Chem. Int. Ed. 57, 626-646 (2018).

76. Dong, Q. et al. Graphene as an intermediary for enhancing the electron transfer rate: a free-standing $\mathrm{Ni}_{3} \mathrm{~S}_{2} @$ graphene@ $\mathrm{Co}_{9} \mathrm{~S}_{8}$ electrocatalytic electrode for oxygen evolution reaction. Nano Res. 11, 1389-1398 (2018).

77. Hong, X., Chan, K., Tsai, C. \& Nørskov, J. K. How doped $\mathrm{MoS}_{2}$ breaks transition-metal scaling relations for $\mathrm{CO}_{2}$ electrochemical reduction. ACS Catal. 6, 4428-4437 (2016).

78. Xing, F. et al. Chemically exfoliated $\mathrm{MoS}_{2}$ for capacitive deionization of saline water. Nano Energy 31, 590-595 (2017).

\section{Acknowledgements}

The authors are grateful to KAUST for very generous financial support.

\section{Author contributions}

P.W. and Y.H. supervised the project; S.Z. conceived the idea and designed the experiments; S.Z. and Y.S. performed the materials synthesis, characterization, and electrochemical measurements; L.L. and D.A. assisted with the TEM characterization; R. L. and L.S. carried out the ICP-AES, BET, Raman, and FTIR measurements; and S.Z. and P.W. co-wrote the paper. All authors discussed and commented on the manuscript.

\section{Additional information}

Supplementary Information accompanies this paper at https://doi.org/10.1038/s41467 018-05474-0.

Competing interests: The authors declare no competing interests.

Reprints and permission information is available online at http://npg.nature.com/ reprintsandpermissions/

Publisher's note: Springer Nature remains neutral with regard to jurisdictional claims in published maps and institutional affiliations.

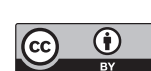

Open Access This article is licensed under a Creative Commons Attribution 4.0 International License, which permits use, sharing, adaptation, distribution and reproduction in any medium or format, as long as you give appropriate credit to the original author(s) and the source, provide a link to the Creative Commons license, and indicate if changes were made. The images or other third party material in this article are included in the article's Creative Commons license, unless indicated otherwise in a credit line to the material. If material is not included in the article's Creative Commons license and your intended use is not permitted by statutory regulation or exceeds the permitted use, you will need to obtain permission directly from the copyright holder. To view a copy of this license, visit http://creativecommons.org/ licenses/by/4.0/

(C) The Author(s) 2018 